\title{
Seasonal Assessment of Chemical and Physical Characterization of Khabur River, Zakho District, Kurdistan Region/Iraq -A Case Study
}

Musher R. A. Al- Barwary ${ }^{a}$

Dept. of Environmental Science, Faculty of Science, University of Zakho, Kurdistan Region, Iraq (musheer.ahmed@uoz.edu.krd)

\begin{abstract}
:
The river Khabur, the life line of more than 500 thousand of people, supplies water for the important requirements of life. In this work, a total of 192 samples of water were collected at Khabur River, Zakho District. The samples were collected monthly at four different seasons like spring, summer, autumn and winter from March, 2019 to February 2020. The samples of water were examined for total hardness (TH), total alkalinity (TA), $\mathrm{pH}, \mathrm{Calcium}\left(\mathrm{Ca}^{2+}\right)$, Electrical conductivity (EC), Nitrate (NO3-), total dissolved solids (TDS), Sulfate $\left(\mathrm{SO}_{4}{ }^{2-}\right)$, phosphate, magnesium $\left(\mathrm{Mg}^{2+}\right) \mathrm{BOD}$, and DO according to the standard methods . The results of the chemical and physical characteristics indicate the water river samples with the following parameters. The total mean values of temperature ranged from $(14.461 \pm 14.1469){ }^{\circ} \mathrm{C}, \mathrm{pH}$ varied from $(7.831 \pm 0.2842)$. Conductivity ranged from $(560.25$ $\pm 110.805) \mu \mathrm{S} / \mathrm{cm})$. Total alkalinity varied from $(194.19 \pm 69.591) \mathrm{mg} / \mathrm{L}$. Total dissolved solids varied from $(355.99 \pm 71.581)$ $\mathrm{mg} / \mathrm{L}$. Dissolved oxygen varied from $(8.219 \pm 0.7989) \mathrm{mg} / \mathrm{L}$. Biochemical oxygen Demand (BOD) ranged from (9.857 \pm 6.0760$)$ $\mathrm{mg} / \mathrm{L}$. Total hardness varied from $(535.02 \pm 78.014) \mathrm{mg} / \mathrm{l}$. Calcium $\left(\mathrm{Ca}^{2+}\right)$ varied from $(409.19 \pm 46.875) \mathrm{mg} / \mathrm{l}$. Magnesium $\left(\mathrm{Mg}^{2+}\right)$ ranged from ( $\left.127.58 \pm 46.875\right) \mathrm{mg} / \mathrm{L}$. Chloride (CL-) varied from ( $\left.26.938 \pm 21.2133\right) \mathrm{mg} / \mathrm{L}$. Nitrate (NO3-) ranged from $(4.449 \pm 2.0327) \mathrm{mg} / \mathrm{L}$. Sulfate $\left(\mathrm{SO}_{4}{ }^{2-}\right)$ varied from $(43.24 \pm 22.479) \mathrm{mg} / \mathrm{l}$. And phosphate ranged from $(4.839 \pm 1.9139) \mu \mathrm{g} / \mathrm{L}$. Gradual increase in total alkalinity and hardness towards the downstream is, due to effluents in the Khabur River. Untreated from sewage discharge on river water it causes of high organic matter, phosphate and Nitrate, at high contents in Khabur River .TDS and EC were found moderate in all locations at Khabur River. The total hardness exceeded the permissible limit in all sites. The $\left(\mathrm{Ca}^{2+}\right)$ and $\left(\mathrm{Mg}^{2+}\right)$ were exceeded the permissible limit in all sites. In the present study an attempt had been made to determine the levels of wastewater that ultimately discharged into Khabor River without proper treatment.
\end{abstract}

KEYWORDS: Waste water, Khabur River, Physical and chemical parameters, Zakho city.

\section{INTRODUCTION}

The Khabur River rises about $100 \mathrm{~km}$ from the city of Zakho, the Khabur River that rises in Turkey and flows through Iraq to meet the Tigris at the tripoint of Iraq, Turkey, and Syria .The Khabur River originates in the Uludere region in turkey and involved a number of small Rivers, flowing off the bolkar mountain and flow southern of Hakkari. The River generally flows south crossing the Iraqi- Turkish border into Kurdistan of Iraq before meeting the Tigris River. Zakho is an important town along the Khabur River where crosses the delal bridge west of Zakho, the small Khabur is joined by its main tributary the Hizil River, which forms part of the border between Iraq and turkey. (Kamil ., 2016). Sources of waste water in the study area are hotels, laundries, industrial wastewater, hospitals, domestic wastewater and household activities. Wastewater is collected through sewage systems. The chemical, biological and physical parameters of Khabur River water are affected by pollutants and affect the quality of water and on water ecosystem. Sediment causes problems by preventing light penetration, covering water organisms, and filling water bodies (Ayoub, et al, 2015). Most of the villagers utilize the river water for domestic purposes. Owing to high values of contaminants like pesticides and toxic metals, the village community establishing on its edge is facing serious health problems. There are hundreds of people suffering from earnest ailments like stomach illness, cancer. The loading of Nitrate and Phosphate on fresh waters promote eutrophication, leading to raising algal blooms and growth of water weeds, due to agricultural seizes contributing nutrients to surface water. (Musher ., 2015) . Excess fertilization leads to variations in water clarity and phytoplankton biomass, which changes the water quality. There are numbers of rural situated on the edge of the Khabur River. The people from these rural utilize the water of Khabur river for livestock rearing and irrigation .This work aims is to evaluate the 13 chemical and physical characteristics of river water in the selected sites of Khabor Rive, Zakho District. And evaluate the effect discharge of wastewater of the Zakho town on chemical and physical, characteristics of Khabur River. And also assessed to determine its suitability for irrigation and drinking purposes

\section{MATERIALS AND METHODS}

2.1 Study area: The river Khabur supplies water for several purposes including domestic, drinking, agriculture, and industrial purpose to number of towns and villages. The untreated wastewater of these villages and cities is being discharged directly in the Khabur River. The studied area are located within Zakho District .The study area falls within Latitude: $37^{\circ} 08^{\prime} 55.36^{\prime \prime} \mathrm{N}$ and Longitude: $42^{\circ} 41^{\prime} 9.28^{\prime \prime} \mathrm{E}$ and lies about $55 \mathrm{~km}$ north of Duhok Governorate .The climate of the study area is tropical summer with extreme temperatures ranging from 47.2 to $15^{\circ} \mathrm{C}$ during summer and winter. (Figure. 1) represents the location of the studied area and sampling sites 


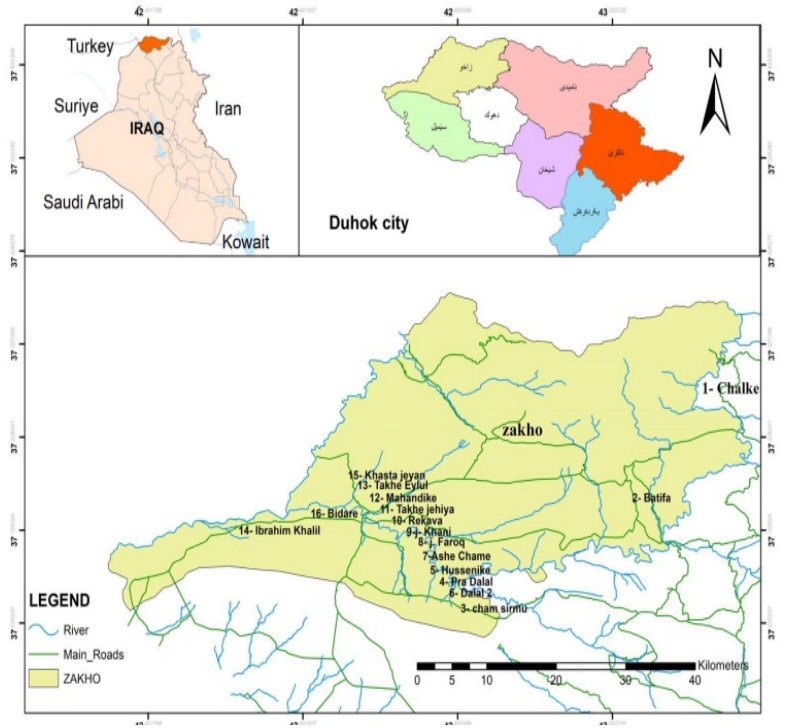

Figure 1: Map of Zakho district showing sampling sites, inflowing Khabur river water

2.2 Samples collection and analysis: Khabur River is located in the northern part of Iraq, for the monthly evaluation the quality of river water. A total of 192 samples of water are collected at Khabur River from various sites of the area study. In the present investigation. River samples are collected at sixteen locations were selected during the period March 2019 to December 2020. Two liters samples were collected in sterilized container, and immediately brought to the laboratory for the chemical and physical tests. The samples of water are tested for the parameters such as total Hardness $(\mathrm{TH}),(\mathrm{Cl})$ Chloride, (TA) Total Alkalinity, Biological Oxygen Demand (BOD), Dissolved Oxygen (DO), (EC), $\mathrm{pH}$, Nitrate $\left(\mathrm{NO}_{3}{ }^{-}\right.$), total Dissolved Solids (TDS), Sulfates $\left(\mathrm{SO}_{4}{ }^{2-}\right.$ ), Electrical
Conductivity, $\left(\mathrm{Mg}^{2+}\right)$ and $\left.\mathrm{Ca}^{2+}\right)$ and phosphates. According to standard methods (APHA., 2017).

Table 1: Monthly variation of Temperature $\left(\mathrm{T}^{\circ} \mathrm{C}\right)$ in sixteen locations

2.3 Statistical Analysis : Statistical analysis of the data was done by analysis of variance which was adopted to analysis the data and LSD0.05 was applied to determine significant differences between periods and hospital using a software program (SPSS version 19). All data were expressed as mean $\pm \mathrm{SE}$.

\section{RESULTS AND DISCUSSION}

Water Temperature $\mathrm{T}^{\circ} \mathrm{C}$ : The temperature of surface water has a direct effect on the biological and chemical processes of the river. It also affects the metabolies of living organisms and physiological processes in the water ecosystem. (MohdYawar et al., 2021). The temperature of Water usually depends on the geographic location, temperature of effluent entering the River and sampling time. (Saif., 2020). The mean value and standard error of mean values were ranged from $(12.075 \pm 3.423)$ to $(24.850 \pm 4.361){ }^{\circ} \mathrm{C}$. (Table 15), The temperature of the collected water samples were in the range $7.2^{\circ} \mathrm{C}$ to $18.9^{\circ} \mathrm{C}$, (Table 1) at all sampling locations in River water (Table1). The River water temperature varied depending on the seasonal variation of atmospheric temperature, it less in winter and higher in summer. The maximum level of sample temperature recorded at site Ashejam was $18.9^{\circ} \mathrm{C}$ in summer, the surface water is influenced by the intensity of the sunlight, while the minimum level was observed at site Jamsemo was $7.2^{\circ} \mathrm{C}$ in February, in winter. Decreasing the temperature of surface water due to the melting of snow of the mountain in the studies area . They have a significant variation at $p \leq 0.05$ ). The same results were observed by (Bishnu ., 2021)

Table 1: Monthly variation of Temperature $\left(\mathrm{T}^{\circ} \mathrm{C}\right)$ in sixteen locations

\begin{tabular}{|c|c|c|c|c|c|c|c|c|c|c|c|c|c|}
\hline $\begin{array}{l}\text { Months } \\
\text { Sites }\end{array}$ & Mar. & Apr. & May. & Jun. & Jul. & Aug. & Sept. & Oct & Nov & Dec & Jan. & Feb & Average \\
\hline 1-Jalke & $14.2 \mathrm{~h}$ & $14.5 \mathrm{~L}$ & $14.8 \mathrm{k}$ & $15.2 \mathrm{j}$ & $15.7 \mathrm{k}$ & $16.3 \mathrm{~L}$ & $11.2 n$ & $10.7 \mathrm{~L}$ & $9.5 \mathrm{~L}$ & $8.4 \mathrm{j}$ & $7.1 \mathrm{k}$ & $7.3 \mathrm{~m}$ & $12.0 \mathrm{a}$ \\
\hline 2-Batefa & $14.1 \mathrm{i}$ & $14.3 \mathrm{~m}$ & $14.9 \mathrm{j}$ & $15.1 \mathrm{k}$ & $15.9 \mathrm{i}$ & $16.2 \mathrm{~m}$ & $11.4 \mathrm{~L}$ & $10.5 \mathrm{~m}$ & $9.4 \mathrm{~m}$ & $8.5 \mathrm{i}$ & $7.7 \mathrm{i}$ & $7.5 \mathrm{~L}$ & $12.1 \mathrm{a}$ \\
\hline 3-Jamsemo & $14.4 \mathrm{f}$ & $14.6 \mathrm{k}$ & $14.7 \mathrm{~L}$ & $15.2 \mathrm{j}$ & $15.8 \mathrm{j}$ & $16.3 \mathrm{~L}$ & $11.3 \mathrm{~m}$ & $10.7 \mathrm{~L}$ & $9.6 \mathrm{k}$ & $8.3 \mathrm{k}$ & $7.9 \mathrm{~g}$ & $7.2 n$ & $12.1 \mathrm{a}$ \\
\hline 4-Aelol Q & $14.3 \mathrm{~g}$ & $14.7 \mathrm{j}$ & $14.9 \mathrm{j}$ & $15.1 \mathrm{k}$ & $15.9 \mathrm{i}$ & $16.3 \mathrm{~L}$ & $11.5 \mathrm{k}$ & $10.9 \mathrm{k}$ & $9.5 \mathrm{~L}$ & $8.5 \mathrm{i}$ & $7,6 j$ & $7.5 \mathrm{~L}$ & $12.2 \mathrm{a}$ \\
\hline 5-Dalal 1 & $14,5 \mathrm{e}$ & $14.9 \mathrm{~h}$ & $15.0 \mathrm{i}$ & $15.3 \mathrm{i}$ & $15.8 \mathrm{j}$ & $16.4 \mathrm{k}$ & $11.7 \mathrm{j}$ & $11,3 \mathrm{j}$ & $9.6 \mathrm{k}$ & $8.7 \mathrm{~g}$ & $7.9 \mathrm{~g}$ & $7.8 \mathrm{j}$ & $11.1 \mathrm{a}$ \\
\hline 6-Dalal 2 & $14.6 \mathrm{~d}$ & $14.8 \mathrm{i}$ & $15.2 \mathrm{~g}$ & $16.3 \mathrm{~g}$ & $16.9 \mathrm{~d}$ & $16,3 \mathrm{~L}$ & $11.9 \mathrm{i}$ & $11.5 \mathrm{i}$ & $9.8 \mathrm{j}$ & $8.9 \mathrm{e}$ & $7.7 \mathrm{i}$ & $7.6 \mathrm{k}$ & $12.6 \mathrm{a}$ \\
\hline 7-Jean & $14.7 \mathrm{c}$ & $14.9 \mathrm{~h}$ & $15.1 \mathrm{~h}$ & $15.5 \mathrm{~h}$ & $16.8 \mathrm{e}$ & $16.5 \mathrm{j}$ & $11.9 \mathrm{i}$ & $11.8 \mathrm{~g}$ & 10. i2 & $8.6 \mathrm{~h}$ & $7.8 \mathrm{~h}$ & $8.0 \mathrm{i}$ & $12.6 \mathrm{a}$ \\
\hline 8-Hasanke & $14.5 \mathrm{e}$ & $15.1 \mathrm{f}$ & $15.3 \mathrm{f}$ & $16.4 \mathrm{f}$ & $15.9 \mathrm{i}$ & $17.4 \mathrm{f}$ & $12.0 \mathrm{~h}$ & $11.9 \mathrm{f}$ & $10.6 \mathrm{~h}$ & $8.8 \mathrm{f}$ & $7.9 \mathrm{~g}$ & $8.2 \mathrm{~g}$ & $14.1 \mathrm{a}$ \\
\hline 9-Rekafa & $14.8 \mathrm{~b}$ & $15.2 \mathrm{e}$ & $15.4 \mathrm{e}$ & $16.7 \mathrm{e}$ & $17.0 \mathrm{c}$ & $17.5 \mathrm{~g}$ & $12.1 \mathrm{~g}$ & $12.2 \mathrm{e}$ & $10.9 \mathrm{~g}$ & $8.9 \mathrm{e}$ & $8.1 \mathrm{f}$ & $8.4 \mathrm{f}$ & $13.1 \mathrm{a}$ \\
\hline 10-Jeous Q & $14.9 \mathrm{a}$ & $15.3 \mathrm{~d}$ & $15.3 \mathrm{f}$ & $1.6 \mathrm{~L}$ & $16.1 \mathrm{~h}$ & $17.6 \mathrm{f}$ & $12.8 \mathrm{e}$ & $12.6 \mathrm{~d}$ & $11.1 \mathrm{f}$ & $9.1 \mathrm{~d}$ & $8.3 \mathrm{e}$ & $8.6 \mathrm{~d}$ & $11.9 \mathrm{a}$ \\
\hline 11-Muhand & $14.8 \mathrm{~b}$ & $15.2 \mathrm{e}$ & $15.5 \mathrm{~d}$ & $17.7 \mathrm{c}$ & $17.1 \mathrm{~b}$ & $18.6 \mathrm{~d}$ & $12.9 \mathrm{~d}$ & $12.7 \mathrm{c}$ & $11.4 \mathrm{e}$ & $9.4 \mathrm{a}$ & $8.9 \mathrm{a}$ & $8.5 \mathrm{e}$ & $13.5 \mathrm{a}$ \\
\hline 12-Farok Br. & $14.9 \mathrm{a}$ & $15.4 \mathrm{c}$ & $15.7 \mathrm{~b}$ & $17.9 \mathrm{a}$ & $16.2 \mathrm{~g}$ & $18.8 \mathrm{~b}$ & $12.9 \mathrm{~d}$ & $12.9 \mathrm{a}$ & $11.6 \mathrm{~d}$ & $9.3 \mathrm{~b}$ & $8.8 \mathrm{~b}$ & $8.7 \mathrm{c}$ & $13.5 \mathrm{a}$ \\
\hline 13-Khane Br. & $14.8 \mathrm{~b}$ & $15.3 \mathrm{~d}$ & $15.6 \mathrm{c}$ & $17.8 \mathrm{~b}$ & $16.1 \mathrm{~h}$ & $18.7 \mathrm{c}$ & $13.0 \mathrm{c}$ & $12.8 \mathrm{~b}$ & $11.7 \mathrm{c}$ & $9.1 \mathrm{~d}$ & $8.7 \mathrm{c}$ & $8.6 \mathrm{~d}$ & $13.5 \mathrm{a}$ \\
\hline 14-Ashe jam & 14.9 & $15.5 \mathrm{~b}$ & $15.7 \mathrm{~b}$ & $17.9 \mathrm{a}$ & $18.2 \mathrm{a}$ & $18.9 \mathrm{a}$ & $13.1 \mathrm{~b}$ & $12.9 \mathrm{a}$ & $11.9 \mathrm{a}$ & $9.2 \mathrm{c}$ & $8.9 \mathrm{a}$ & $8.8 \mathrm{~b}$ & $13.8 \mathrm{a}$ \\
\hline 15-Bedara & $14.8 \mathrm{a}$ & $15.6 \mathrm{a}$ & $15.8 \mathrm{a}$ & $17.0 \mathrm{~d}$ & $16.4 \mathrm{f}$ & $18.3 \mathrm{e}$ & $13.3 \mathrm{a}$ & $12.8 \mathrm{~b}$ & $11.8 \mathrm{~b}$ & $8.7 \mathrm{~g}$ & $8.8 \mathrm{~b}$ & $8.9 \mathrm{a}$ & $13.5 \mathrm{a}$ \\
\hline 16-Ibrhem Br. & $14.4 \mathrm{f}$ & $15.0 \mathrm{~g}$ & $15.2 \mathrm{~g}$ & $16.4 \mathrm{f}$ & $15.8 \mathrm{j}$ & $16.7 \mathrm{i}$ & $12.2 \mathrm{f}$ & $11.6 \mathrm{~h}$ & $10.9 \mathrm{~g}$ & $8.9 \mathrm{e}$ & $8.6 \mathrm{~d}$ & $8.1 \mathrm{~h}$ & $12.8 \mathrm{a}$ \\
\hline
\end{tabular}

EC) Electrical Conductivity $\mu \mathrm{S} / \mathrm{cm})$ : In this study. The mean value and standard error of mean values were ranged from $(446.75 \pm 36.544)$ to $(675.00 \pm 91.254) \mu \mathrm{S} / \mathrm{cm})$, (Table 15) .The total mean value of (EC) $(560.25 \mu \mathrm{S} / \mathrm{cm})$ are within the safe level, according to (WHO., 2012) was $1000 \mu \mathrm{S} / \mathrm{cm}$, for drinking uses . From the results shown in (Table 2), The value of Electrical Conductivity in this study showed a range of (441 $\mu \mathrm{S} / \mathrm{cm}$ to $863 \mu \mathrm{S} / \mathrm{cm})$. The value of conductivity was recorded lowest in Batefa site was $411 \mu \mathrm{S} / \mathrm{cm}$ in August, and the maximum

Table 2 Monthly variation of (EC) Electrical Conductivity $(\mu \mathrm{S} / \mathrm{cm})$

\begin{tabular}{|c|c|c|c|c|c|c|c|c|c|c|c|c|c|}
\hline $\begin{array}{l}\text { Months } \\
\text { Sites }\end{array}$ & Mar. & Apr. & May. & Jun. & Jul. & Aug. & Sept. & Oct & Nov & Dec & Jan. & $\mathrm{Feb}$ & Average \\
\hline 1-Jalke & $417 p$ & $473 \mathrm{o}$ & $458 \mathrm{n}$ & $431 \mathrm{n}$ & $417 p$ & $402 p$ & $429 \mathrm{o}$ & $435 \mathrm{~L}$ & $429 \mathrm{j}$ & $463 p$ & $469 \mathrm{~m}$ & 5380 & $446 \mathrm{e}$ \\
\hline 2-Batefa & 4250 & $485 n$ & $462 \mathrm{~m}$ & $442 \mathrm{~L}$ & $426 \mathrm{~m}$ & 4110 & $449 n$ & $427 \mathrm{o}$ & $418 \mathrm{~m}$ & $485 n$ & 4580 & $541 \mathrm{n}$ & $452 \mathrm{e}$ \\
\hline 3-Jamsemo & $438 n$ & $469 p$ & 449 o & $463 \mathrm{~h}$ & $419 \mathrm{o}$ & $426 n$ & $453 m$ & $429 n$ & $420 \mathrm{k}$ & $488 \mathrm{~m}$ & $463 n$ & $529 p$ & $453 \mathrm{e}$ \\
\hline
\end{tabular}

conductivity were recorded in site bedara in February was 863 $\mu \mathrm{S} / \mathrm{cm}$. (EC) in all seasons was highest in winter. The high conductivity is likely due to extensive agricultural practices and geological conditions. The concentrations of all sampling sites were within the standard concentration of (WHO, 2012). The studied River water has significant variation in (EC) of the study times (Table- 15 ). It has asignificant differences at $p \leq$ 0.05 ) (Table2). In this paper are significantly minimum than those obtained by (Lilia .,.2020) 


\begin{tabular}{|l|l|l|l|l|l|l|l|l|l|l|l|l|l|}
\hline 4-Aelol Q & $457 \mathrm{~m}$ & $492 \mathrm{~m}$ & $463 \mathrm{~L}$ & $459 \mathrm{j}$ & $420 \mathrm{n}$ & $448 \mathrm{~m}$ & $461 \mathrm{~L}$ & $433 \mathrm{~m}$ & $429 \mathrm{j}$ & $479 \mathrm{o}$ & $449 \mathrm{p}$ & $569 \mathrm{~m}$ & $463 \mathrm{e}$ \\
\hline 5-Dalal 1 & $466 \mathrm{~L}$ & $538 \mathrm{~L}$ & $458 \mathrm{n}$ & $442 \mathrm{~L}$ & $462 \mathrm{~L}$ & $484 \mathrm{~L}$ & $489 \mathrm{k}$ & $421 \mathrm{p}$ & $431 \mathrm{i}$ & $518 \mathrm{~L}$ & $470 \mathrm{~L}$ & $583 \mathrm{~L}$ & $480 \mathrm{e}$ \\
\hline 6-Dalal 2 & $479 \mathrm{k}$ & $553 \mathrm{k}$ & $474 \mathrm{k}$ & $459 \mathrm{j}$ & $577 \mathrm{~g}$ & $569 \mathrm{i}$ & $513 \mathrm{j}$ & $552 \mathrm{i}$ & $429 \mathrm{j}$ & $536 \mathrm{k}$ & $479 \mathrm{j}$ & $619 \mathrm{k}$ & $519 \mathrm{~d}$ \\
\hline 7-Jean & $481 \mathrm{j}$ & $581 \mathrm{j}$ & $481 \mathrm{j}$ & $437 \mathrm{~m}$ & $559 \mathrm{i}$ & $511 \mathrm{k}$ & $533 \mathrm{i}$ & $549 \mathrm{j}$ & $431 \mathrm{i}$ & $594 \mathrm{j}$ & $486 \mathrm{i}$ & $636 \mathrm{j}$ & $475 \mathrm{~d}$ \\
\hline 8-Hasanke & $519 \mathrm{i}$ & $629 \mathrm{i}$ & $517 \mathrm{i}$ & $471 \mathrm{f}$ & $513 \mathrm{k}$ & $530 \mathrm{j}$ & $559 \mathrm{~h}$ & $563 \mathrm{~h}$ & $419 \mathrm{~L}$ & $621 \mathrm{i}$ & $473 \mathrm{k}$ & $695 \mathrm{i}$ & $585 \mathrm{~d}$ \\
\hline 9-Rekafa & $537 \mathrm{~g}$ & $741 \mathrm{~d}$ & $531 \mathrm{~h}$ & $449 \mathrm{k}$ & $532 \mathrm{j}$ & $596 \mathrm{~h}$ & $574 \mathrm{f}$ & $772 \mathrm{a}$ & $439 \mathrm{~h}$ & $643 \mathrm{~h}$ & $490 \mathrm{~h}$ & $717 \mathrm{~h}$ & $686 \mathrm{c}$ \\
\hline 10-Jeous Q & $526 \mathrm{~h}$ & $694 \mathrm{f}$ & $544 \mathrm{~g}$ & $468 \mathrm{~g}$ & $586 \mathrm{f}$ & $625 \mathrm{~g}$ & $563 \mathrm{~g}$ & $658 \mathrm{f}$ & $462 \mathrm{~g}$ & $660 \mathrm{f}$ & $510 \mathrm{~g}$ & $738 \mathrm{~g}$ & $639 \mathrm{c}$ \\
\hline 11-Muhand & $684 \mathrm{~b}$ & $713 \mathrm{e}$ & $593 \mathrm{f}$ & $460 \mathrm{i}$ & $653 \mathrm{c}$ & $651 \mathrm{f}$ & $574 \mathrm{f}$ & $663 \mathrm{e}$ & $477 \mathrm{f}$ & $729 \mathrm{c}$ & $539 \mathrm{f}$ & $816 \mathrm{f}$ & $658 \mathrm{~b}$ \\
\hline 12-Farok B & $539 \mathrm{f}$ & $686 \mathrm{~g}$ & $627 \mathrm{e}$ & $511 \mathrm{e}$ & $670 \mathrm{~b}$ & $664 \mathrm{e}$ & $612 \mathrm{e}$ & $769 \mathrm{~b}$ & $486 \mathrm{e}$ & $719 \mathrm{~d}$ & $557 \mathrm{e}$ & $835 \mathrm{e}$ & $675 \mathrm{ab}$ \\
\hline 13-Khane B & $572 \mathrm{e}$ & $742 \mathrm{c}$ & $671 \mathrm{~b}$ & $539 \mathrm{~d}$ & $694 \mathrm{a}$ & $699 \mathrm{~b}$ & $639 \mathrm{~d}$ & $675 \mathrm{~d}$ & $497 \mathrm{~d}$ & $731 \mathrm{~b}$ & $593 \mathrm{~d}$ & $844 \mathrm{c}$ & $658 \mathrm{ab}$ \\
\hline 14-Ashe jam & $649 \mathrm{~d}$ & $761 \mathrm{~b}$ & $690 \mathrm{a}$ & $577 \mathrm{c}$ & $618 \mathrm{e}$ & $736 \mathrm{a}$ & $661 \mathrm{c}$ & $683 \mathrm{c}$ & $514 \mathrm{c}$ & $749 \mathrm{a}$ & $611 \mathrm{c}$ & $851 \mathrm{~b}$ & $675 \mathrm{a}$ \\
\hline 15-Bedara & $661 \mathrm{c}$ & $775 \mathrm{a}$ & $649 \mathrm{c}$ & $593 \mathrm{~b}$ & $633 \mathrm{~d}$ & $670 \mathrm{~d}$ & $683 \mathrm{~b}$ & $591 \mathrm{~g}$ & $522 \mathrm{~b}$ & $650 \mathrm{~g}$ & $627 \mathrm{~b}$ & $863 \mathrm{a}$ & $659 \mathrm{ab}$ \\
\hline 16-Ibrhem B & $693 \mathrm{a}$ & $659 \mathrm{~h}$ & $638 \mathrm{~d}$ & $610 \mathrm{a}$ & $569 \mathrm{~h}$ & $683 \mathrm{c}$ & $711 \mathrm{a}$ & $527 \mathrm{k}$ & $558 \mathrm{a}$ & $669 \mathrm{e}$ & $631 \mathrm{a}$ & $841 \mathrm{~d}$ & $649 \mathrm{ab}$ \\
\hline
\end{tabular}

Total Dissolved Solid (TDS) in mg/L : Total dissolved solid in all water samples found majority in the form of potassium, calcium, sodium, carbonates , chlorides, magnesium, bicarbonates, sulfates and nitrates. They affect the corrosive and water hardness (MohdYawar et al., 2021). In the present investigation.The mean and standard error values were ranged from $(285.42 \pm 23.500)$ to $(424.08 \pm 66.432) \mathrm{mg} / \mathrm{L}$. (Table 15), The total mean value of (TDS) $(355.99 \mathrm{mg} / \mathrm{L})$ is within the safe level, according to (WHO., 2012). The (TDS) ranged between 266 and $545 \mathrm{mg} / 1$ (Table 3).The high concentration of (TDS) found at site Farok Bridge in February was $545 \mathrm{mg} / \mathrm{L}$, in the winter season. The presence of high concentration of (TDS)

Table 3 Monthly variation of (TDS) Total dissolved solids ( $\mathrm{mg} / \mathrm{L}$ ) in sixteen location

\begin{tabular}{|c|c|c|c|c|c|c|c|c|c|c|c|c|c|}
\hline Months & Mar. & Apr. & May. & Jun. & Jul. & Aug. & Sept. & Oct. & Nov. & Dec. & Jan. & Feb. & Average \\
\hline 1-Jalke & 2660 & $302 \mathrm{~m}$ & $293 m$ & $275 \mathrm{~m}$ & $266 n$ & $257 \mathrm{n}$ & $274 \mathrm{o}$ & $278 \mathrm{j}$ & $274 \mathrm{i}$ & 2960 & $300 \mathrm{~L}$ & $344 n$ & $285 \mathrm{e}$ \\
\hline 2-Batefa & $272 n$ & $310 \mathrm{~L}$ & $295 \mathrm{~L}$ & $282 \mathrm{k}$ & $272 \mathrm{~L}$ & $263 \mathrm{~m}$ & $287 n$ & $273 \mathrm{~m}$ & $267 \mathrm{k}$ & $310 \mathrm{~m}$ & $293 m$ & $346 \mathrm{~m}$ & $289 \mathrm{e}$ \\
\hline 3-Jamsemo & $280 \mathrm{~m}$ & $300 \mathrm{n}$ & $287 \mathrm{n}$ & $296 \mathrm{~h}$ & $268 \mathrm{~m}$ & $227 \mathrm{o}$ & $289 \mathrm{~m}$ & $274 \mathrm{~L}$ & $268 \mathrm{j}$ & $312 \mathrm{~L}$ & $496 a$ & 338 o & $289 \mathrm{e}$ \\
\hline 4-Aelol Q & $292 \mathrm{~L}$ & $314 \mathrm{k}$ & $296 \mathrm{k}$ & $293 \mathrm{j}$ & $268 \mathrm{~m}$ & $286 \mathrm{~L}$ & $295 \mathrm{~L}$ & $277 \mathrm{k}$ & $274 i$ & $306 n$ & $287 n$ & $364 \mathrm{~L}$ & $296 \mathrm{e}$ \\
\hline 5-Dalal 1 & $298 \mathrm{k}$ & $344 \mathrm{i}$ & $293 \mathrm{~m}$ & $282 \mathrm{k}$ & $295 \mathrm{k}$ & $309 \mathrm{k}$ & $312 \mathrm{k}$ & $269 n$ & $257 \mathrm{~L}$ & $331 \mathrm{j}$ & $300 \mathrm{~L}$ & $373 \mathrm{k}$ & $307 \mathrm{e}$ \\
\hline 6-Dalal 2 & $306 \mathrm{j}$ & $353 \mathrm{~h}$ & $303 \mathrm{j}$ & $293 j$ & $369 \mathrm{~g}$ & $364 \mathrm{~h}$ & $328 \mathrm{j}$ & $353 \mathrm{~g}$ & $274 \mathrm{i}$ & $343 \mathrm{i}$ & $306 \mathrm{j}$ & $396 \mathrm{j}$ & $332 d$ \\
\hline 7-Jean & $307 \mathrm{i}$ & $371 \mathrm{~g}$ & $307 i$ & $279 \mathrm{~L}$ & $357 \mathrm{~h}$ & $327 \mathrm{j}$ & $341 \mathrm{i}$ & $351 \mathrm{~h}$ & $275 \mathrm{~h}$ & $380 \mathrm{~h}$ & $311 \mathrm{i}$ & $407 \mathrm{i}$ & $303 \mathrm{~d}$ \\
\hline 8-Hasanke & $332 \mathrm{~h}$ & $402 \mathrm{f}$ & $330 \mathrm{~h}$ & $301 \mathrm{f}$ & $328 \mathrm{j}$ & $339 \mathrm{i}$ & $357 \mathrm{~h}$ & $360 \mathrm{f}$ & $268 \mathrm{j}$ & $397 \mathrm{f}$ & $302 \mathrm{k}$ & $444 \mathrm{~g}$ & $346 \mathrm{~d}$ \\
\hline 9-Rekafa & $343 \mathrm{f}$ & $474 \mathrm{c}$ & $339 \mathrm{~g}$ & $319 \mathrm{e}$ & $340 \mathrm{i}$ & $381 \mathrm{~g}$ & $368 \mathrm{e}$ & $494 \mathrm{a}$ & $280 \mathrm{~g}$ & $411 \mathrm{e}$ & $313 \mathrm{~h}$ & $458 \mathrm{f}$ & $374 \mathrm{c}$ \\
\hline 10-Jeous Q & $336 \mathrm{~g}$ & $444 \mathrm{e}$ & $348 \mathrm{f}$ & $299 \mathrm{~g}$ & $375 f$ & $400 \mathrm{f}$ & $360 \mathrm{~g}$ & $421 \mathrm{e}$ & $495 \mathrm{a}$ & $422 d$ & $326 \mathrm{~g}$ & $427 \mathrm{~h}$ & $375 \mathrm{bc}$ \\
\hline 11-Muhand & $437 \mathrm{a}$ & $458 \mathrm{~d}$ & $397 \mathrm{e}$ & $294 \mathrm{i}$ & $417 \mathrm{c}$ & $416 \mathrm{e}$ & $367 \mathrm{f}$ & $224 \mathrm{o}$ & $305 \mathrm{f}$ & $466 \mathrm{~b}$ & $344 f$ & $522 \mathrm{e}$ & $387 \mathrm{bc}$ \\
\hline 12-Farok Br & $344 \mathrm{e}$ & $339 \mathrm{j}$ & $401 d$ & $327 d$ & $428 b$ & $424 d$ & $391 d$ & $492 b$ & $311 d$ & $460 c$ & $356 \mathrm{e}$ & $545 \mathrm{~b}$ & $408 \mathrm{abc}$ \\
\hline 13-Khane $\mathrm{Br}$ & $366 \mathrm{~d}$ & $474 \mathrm{c}$ & $429 b$ & $344 c$ & $444 a$ & $447 b$ & $408 \mathrm{c}$ & $432 d$ & $306 \mathrm{e}$ & $394 \mathrm{~g}$ & $379 d$ & $540 \mathrm{~d}$ & $421 \mathrm{ab}$ \\
\hline 14-Ashe jam & $415 \mathrm{c}$ & $487 \mathrm{~b}$ & $441 \mathrm{a}$ & $361 b$ & $395 \mathrm{e}$ & $488 \mathrm{a}$ & $423 b$ & $337 \mathrm{i}$ & $328 \mathrm{c}$ & $479 a$ & $391 \mathrm{c}$ & $544 \mathrm{c}$ & $432 \mathrm{a}$ \\
\hline 15-Bedara & $423 \mathrm{~b}$ & $496 \mathrm{a}$ & $415 c$ & $379 a$ & $405 d$ & $428 \mathrm{c}$ & $437 \mathrm{a}$ & $478 \mathrm{c}$ & $334 b$ & $316 \mathrm{k}$ & $401 \mathrm{~b}$ & $552 \mathrm{a}$ & $421 \mathrm{a}$ \\
\hline 16- IbrahemBr. & $415 c$ & $474 c$ & $397 \mathrm{e}$ & $327 d$ & $357 f$ & $381 \mathrm{~g}$ & $423 b$ & $338 \mathrm{i}$ & $328 \mathrm{c}$ & $314 \mathrm{k}$ & $391 \mathrm{c}$ & $540 \mathrm{~d}$ & $421 \mathrm{ab}$ \\
\hline
\end{tabular}

$\mathrm{pH}$ (Table.4) explain the $\mathrm{pH}$ values at sixteen different locations of the Khabur River. It observed that $\mathrm{pH}$ concentration of water samples was ranged from 7.3 to 8.4, depending on the site. The high values of $\mathrm{pH}$ in the river water may be due to the discharge of wastewater, agriculture and industries in to the Khabur River, which increases the $\mathrm{pH}$ of river water. The mean value and standard error of mean values were ranged from $(7.517 \pm 2167)$ to $(8.142 \pm 0.2065)$ (Table 15). The total mean concentrations of $(\mathrm{pH})$ were in the safe Table 4 Monthly variation of $\mathrm{pH}$ value in sixteen locations

\begin{tabular}{|l|l|l|l|l|l|l|l|l|l|l|l|l|l|}
\hline $\begin{array}{c}\text { Months } \\
\text { Sites }\end{array}$ & Mar. & Apr. & May. & Jun & Jul. & Aug. & Sept & Oct. & Nov. & Dec & Jan. & Feb & Average \\
\hline 1-Jalke & $7.8 \mathrm{c}$ & $8.1 \mathrm{~b}$ & $7.9 \mathrm{~b}$ & $8.3 \mathrm{c}$ & $8.0 \mathrm{~d}$ & $8.1 \mathrm{c}$ & $8.2 \mathrm{a}$ & $7.8 \mathrm{~d}$ & $8.0 \mathrm{c}$ & $8.1 \mathrm{~b}$ & $8.3 \mathrm{~b}$ & $8.2 \mathrm{~b}$ & $8.0 \mathrm{ab}$ \\
\hline 2-Batefa & $7.9 \mathrm{~b}$ & $8.2 \mathrm{a}$ & $7.7 \mathrm{~d}$ & $8.5 \mathrm{a}$ & $8.2 \mathrm{~b}$ & $8.3 \mathrm{a}$ & $8.0 \mathrm{c}$ & $8.1 \mathrm{a}$ & $8.2 \mathrm{a}$ & $8.2 \mathrm{a}$ & $8.1 \mathrm{~d}$ & $8.3 \mathrm{a}$ & $8.1 \mathrm{a}$ \\
\hline 3-Jamsemo & $7.8 \mathrm{c}$ & $8.0 \mathrm{c}$ & $7.8 \mathrm{c}$ & $8.1 \mathrm{~d}$ & $8.1 \mathrm{c}$ & $8.0 \mathrm{~d}$ & $8.1 \mathrm{~b}$ & $7.9 \mathrm{c}$ & $8.0 \mathrm{c}$ & $8.0 \mathrm{c}$ & $8.2 \mathrm{c}$ & $8.2 \mathrm{~b}$ & $8.0 \mathrm{bc}$ \\
\hline 4-Aelol Q & $7.6 \mathrm{e}$ & $7.9 \mathrm{~d}$ & $7.9 \mathrm{~b}$ & $8.4 \mathrm{~b}$ & $8.3 \mathrm{a}$ & $8.2 \mathrm{~b}$ & $7.9 \mathrm{~d}$ & $7.8 \mathrm{~d}$ & $8.1 \mathrm{~b}$ & $8.2 \mathrm{a}$ & $8.0 \mathrm{e}$ & $8.1 \mathrm{c}$ & $8.0 \mathrm{bc}$ \\
\hline 5-Dalal 1 & $7.7 \mathrm{~d}$ & $7.8 \mathrm{e}$ & $8.1 \mathrm{a}$ & $8.0 \mathrm{e}$ & $8.1 \mathrm{c}$ & $8.0 \mathrm{~d}$ & $7.7 \mathrm{f}$ & $8.0 \mathrm{~b}$ & $7.8 \mathrm{e}$ & $8.1 \mathrm{~b}$ & $8.3 \mathrm{~b}$ & $8.2 \mathrm{~b}$ & $7.9 \mathrm{bcd}$ \\
\hline 6-Dalal 2 & $7.5 \mathrm{f}$ & $7.4 \mathrm{i}$ & $7.7 \mathrm{~d}$ & $7.8 \mathrm{f}$ & $8.0 \mathrm{~d}$ & $8.2 \mathrm{~b}$ & $8.0 \mathrm{c}$ & $7.9 \mathrm{c}$ & $8.0 \mathrm{c}$ & $8.1 \mathrm{~b}$ & $8.2 \mathrm{c}$ & $8.3 \mathrm{a}$ & $7.9 \mathrm{cde}$ \\
\hline 7-Jean & $7.6 \mathrm{e}$ & $7.7 \mathrm{f}$ & $7.9 \mathrm{~b}$ & $7.4 \mathrm{j}$ & $7.7 \mathrm{~g}$ & $7.6 \mathrm{~g}$ & $7,8 \mathrm{e}$ & $8.1 \mathrm{a}$ & $7.7 \mathrm{f}$ & $8.0 \mathrm{c}$ & $8.3 \mathrm{~b}$ & $8.0 \mathrm{~d}$ & $7.8 \mathrm{efg}$ \\
\hline 8-Hasanke & $7.7 \mathrm{~d}$ & $7.5 \mathrm{~h}$ & $7.8 \mathrm{c}$ & $7.6 \mathrm{~h}$ & $7.9 \mathrm{e}$ & $7.7 \mathrm{f}$ & $7.6 \mathrm{~g}$ & $7.7 \mathrm{e}$ & $7.9 \mathrm{~d}$ & $8.1 \mathrm{~b}$ & $8.1 \mathrm{~d}$ & $8.2 \mathrm{~b}$ & $7.8 \mathrm{efg}$ \\
\hline 9-Rekafa & $7.5 \mathrm{f}$ & $7.7 \mathrm{f}$ & $7.5 \mathrm{f}$ & $7.7 \mathrm{~g}$ & $7.6 \mathrm{~h}$ & $7.5 \mathrm{~h}$ & $7.8 \mathrm{e}$ & $7.5 \mathrm{f}$ & $7.6 \mathrm{~g}$ & $7.8 \mathrm{e}$ & $8.4 \mathrm{a}$ & $8.1 \mathrm{c}$ & $7.7 \mathrm{ghi}$ \\
\hline 10-Jeous Q & $8.1 \mathrm{a}$ & $7.6 \mathrm{~g}$ & $7.6 \mathrm{e}$ & $7.5 \mathrm{i}$ & $7.8 \mathrm{f}$ & $7.7 \mathrm{f}$ & $7.4 \mathrm{i}$ & $7.9 \mathrm{c}$ & $7.8 \mathrm{e}$ & $7.9 \mathrm{~d}$ & $8.1 \mathrm{~d}$ & $8.2 \mathrm{~b}$ & $7.8 \mathrm{fg}$ \\
\hline 11-Muhand & $7.4 \mathrm{~g}$ & $7.4 \mathrm{i}$ & $7.5 \mathrm{f}$ & $7.4 \mathrm{j}$ & $7.7 \mathrm{~g}$ & $7.9 \mathrm{e}$ & $7.7 \mathrm{f}$ & $7.5 \mathrm{f}$ & $7.4 \mathrm{~h}$ & $7.7 \mathrm{f}$ & $8.2 \mathrm{c}$ & $8.0 \mathrm{~d}$ & $7.6 \mathrm{hi}$ \\
\hline
\end{tabular}

limit (6.5-8.5).The standard concentration of $\mathrm{pH}$ for drinking water was (6.5-8.5). Set by (WHO , 2011). In this work, the maximum concentration was recorded in site Rekafa in January in winter was (8.4). The higher $\mathrm{pH}$ values are due to bicarbonate, carbon dioxide and carbonate. While the minimum value was recorded in Ashjame in August was (7.3). The studied River water has significant variation in $(\mathrm{pH})$ at the study times. They have a significant Same results obtained by (O.I.Solana1et.al.,2020). may be due to the influence of agricultural activities, domestic sewage, and solid waste dumping. The minimum range of TDS was seen at location of Jalke in March was $266 \mathrm{mg} / \mathrm{L}$. in the spring season. The safe value of total dissolved solid for drinking water is $500 \mathrm{mg} / \mathrm{l}$. TDS content in most samples were within the safe range of $500 \mathrm{mg} / \mathrm{L}$, of (WHO, 2012) .The studied River water has significant variation in (TDS) along the study periods. They have a significant variation at $\mathrm{p} \leq 0.05$ ). (Table15). This work are significantly higher than those obtained by (Bishnu., 2021). Because of geological formation in the study area. 


\begin{tabular}{|l|l|l|l|l|l|l|l|l|l|l|l|l|l|}
\hline $\begin{array}{l}\text { 12-Farok } \\
\mathrm{Br}\end{array}$ & $7.7 \mathrm{~d}$ & $7.6 \mathrm{~g}$ & $7.7 \mathrm{~d}$ & $7.5 \mathrm{i}$ & $7.9 \mathrm{e}$ & $7.5 \mathrm{~h}$ & $7.4 \mathrm{i}$ & $7.8 \mathrm{~d}$ & $7.7 \mathrm{f}$ & $7.9 \mathrm{~d}$ & $8.0 \mathrm{e}$ & $8.2 \mathrm{~b}$ & $7.7 \mathrm{gh}$ \\
\hline $\begin{array}{c}13-\text { Khane } \\
\mathrm{Br}\end{array}$ & $7.4 \mathrm{~g}$ & $7.5 \mathrm{~h}$ & $7.6 \mathrm{e}$ & $7.2 \mathrm{~L}$ & $7.5 \mathrm{i}$ & $7.7 \mathrm{f}$ & $7.6 \mathrm{~g}$ & $7.7 \mathrm{e}$ & $7.6 \mathrm{~g}$ & $7.8 \mathrm{e}$ & $8.1 \mathrm{~d}$ & $7.8 \mathrm{e}$ & $7.6 \mathrm{jj}$ \\
\hline $\begin{array}{c}14-\text { Ashe } \\
\text { jam }\end{array}$ & $7.4 \mathrm{~g}$ & $7.4 \mathrm{i}$ & $7.3 \mathrm{~h}$ & $7.6 \mathrm{~h}$ & $7.7 \mathrm{~g}$ & $7.3 \mathrm{i}$ & $7.3 \mathrm{j}$ & $7.5 \mathrm{f}$ & $7.3 \mathrm{i}$ & $7.7 \mathrm{f}$ & $7.9 \mathrm{f}$ & $7.8 \mathrm{e}$ & $7.5 \mathrm{k}$ \\
\hline $15-$-Bedara & $7.5 \mathrm{f}$ & $7.8 \mathrm{e}$ & $7.4 \mathrm{~g}$ & $7.3 \mathrm{k}$ & $7.4 \mathrm{j}$ & $7.7 \mathrm{f}$ & $7.5 \mathrm{~h}$ & $7.7 \mathrm{e}$ & $7.1 \mathrm{j}$ & $7.8 \mathrm{e}$ & $7.6 \mathrm{~g}$ & $7.7 \mathrm{f}$ & $7.5 \mathrm{jk}$ \\
\hline $\begin{array}{l}16-\mathrm{Ibrhem} \\
\mathrm{Br}\end{array}$ & $7.7 \mathrm{~d}$ & $8.1 \mathrm{~b}$ & $7.8 \mathrm{c}$ & $7.6 \mathrm{~h}$ & $7.8 \mathrm{f}$ & $8.0 \mathrm{~d}$ & $7.9 \mathrm{~d}$ & $8.1 \mathrm{a}$ & $7.7 \mathrm{f}$ & $7.9 \mathrm{~d}$ & $8.0 \mathrm{e}$ & $8.1 \mathrm{c}$ & $7.8 \mathrm{def}$ \\
\hline
\end{tabular}

Dissolved oxygen (DO) in $\mathrm{mg} / \mathrm{L}$ : The decomposition and oxidation of organic material reduce the solubility of oxygen in the water. DO is a major parameter of water quality evaluation and biological processes in river water (Saurabh et al ., 2021). The means value and standard error of mean values were ranged from $(7.358 \pm 0.6417)$ to $(8.942 \pm 0.5760) \mathrm{mg} / \mathrm{L}$, (Table 15). The total mean value of (DO) were within the permissible limit. An ideal DO concentration of $5.0 \mathrm{mg} / \mathrm{L}$ is the standard for domestic water (Ackson ., 2020). In this work DO range from $6.6 \mathrm{mg} / \mathrm{l}$ to $9.9 \mathrm{mg} / \mathrm{L}$, (Table 5). The minimum value recorded during summer at site Muhand in August was 6.6 $\mathrm{mg} / \mathrm{L}$, The lowest concentration of DO may be the cause of high temperature and addition of wastewater to Khabur River. Higher concentration of (DO) indicates good water life. The studied River water has significant variation in (DO) at the study times. They have a significant differences at $\mathrm{p} \leq 0.05$ ). (Table2). This work are significantly maximum than those obtained by (NidhiGupta et al .,2017).

\begin{tabular}{|c|c|c|c|c|c|c|c|c|c|c|c|c|c|}
\hline $\begin{array}{ll}\text { Months } \\
\text { Sites }\end{array}$ & Mar. & Apr. & May. & Jun. & Jul. & Aug. & Sept. & Oct. & Nov. & Dec. & Jan. & Feb. & Average \\
\hline 1-Jalke & $9.9 \mathrm{a}$ & $8.6 \mathrm{e}$ & $9.7 \mathrm{~b}$ & $8.2 \mathrm{c}$ & $8.7 \mathrm{a}$ & $8.4 \mathrm{c}$ & $8.0 \mathrm{~b}$ & $8.5 \mathrm{~b}$ & $8.3 \mathrm{~d}$ & $9.5 \mathrm{~d}$ & $9.3 b$ & $9.7 \mathrm{~b}$ & $8.9 \mathrm{ab}$ \\
\hline 2-Batefa & $9.4 \mathrm{~b}$ & $8.9 \mathrm{~b}$ & $9.7 \mathrm{~b}$ & $8.5 \mathrm{a}$ & $8.3 \mathrm{~d}$ & $8.7 \mathrm{~b}$ & $7.8 \mathrm{~d}$ & $8.9 \mathrm{a}$ & $8.9 \mathrm{a}$ & $9.7 \mathrm{~b}$ & $9.0 \mathrm{c}$ & $9.5 \mathrm{~d}$ & $8.9 \mathrm{a}$ \\
\hline 3-Jamsemo & $8.7 \mathrm{e}$ & $9.1 \mathrm{a}$ & $9.4 \mathrm{~d}$ & $8.1 \mathrm{~d}$ & $8.6 \mathrm{~b}$ & $8.9 \mathrm{a}$ & $7.4 \mathrm{~g}$ & $8.3 \mathrm{c}$ & $8.6 \mathrm{c}$ & $9.9 \mathrm{a}$ & $9.6 \mathrm{a}$ & $9.8 \mathrm{a}$ & $8.8 \mathrm{ab}$ \\
\hline 4-Aelol Q & $8.8 \mathrm{~d}$ & $8.9 \mathrm{~b}$ & $9.6 \mathrm{c}$ & $8.0 \mathrm{e}$ & $8.4 \mathrm{c}$ & $8.3 \mathrm{~d}$ & $8.1 \mathrm{a}$ & $8.5 \mathrm{~b}$ & $8.8 \mathrm{~b}$ & $9.6 \mathrm{c}$ & $9.3 \mathrm{~b}$ & $9.6 \mathrm{c}$ & $8.8 \mathrm{ab}$ \\
\hline 5-Dalal 1 & $9.3 \mathrm{c}$ & $9.1 \mathrm{a}$ & $9.8 \mathrm{a}$ & $8.4 \mathrm{~b}$ & $8,1 \mathrm{e}$ & $8.0 \mathrm{f}$ & $7.9 \mathrm{c}$ & $8.9 \mathrm{a}$ & $8.3 \mathrm{~d}$ & $9.3 \mathrm{e}$ & $9.0 \mathrm{c}$ & $9.6 \mathrm{c}$ & $8.8 \mathrm{ab}$ \\
\hline 6-Dalal 2 & $9.3 \mathrm{c}$ & $8.8 \mathrm{c}$ & $9.4 \mathrm{~d}$ & $8.1 \mathrm{~d}$ & $7.9 \mathrm{~g}$ & $8.3 \mathrm{~d}$ & $7.7 \mathrm{e}$ & $8.3 \mathrm{c}$ & $8.0 \mathrm{f}$ & $9.0 \mathrm{f}$ & $8.9 \mathrm{~d}$ & $9.7 \mathrm{~b}$ & $8.6 \mathrm{~b}$ \\
\hline 7-Jean & $8.5 \mathrm{f}$ & $8.6 \mathrm{e}$ & $9.1 \mathrm{f}$ & $7.8 \mathrm{~g}$ & $7.5 \mathrm{~h}$ & $8.1 \mathrm{e}$ & $7.4 \mathrm{~g}$ & $8.1 \mathrm{e}$ & $8.3 \mathrm{~d}$ & $8.8 \mathrm{~g}$ & $8.5 \mathrm{~g}$ & $8.9 \mathrm{e}$ & $8.3 \mathrm{c}$ \\
\hline 8-Hasanke & $8.2 \mathrm{~g}$ & $8.7 \mathrm{~d}$ & $9.3 \mathrm{e}$ & $8.0 \mathrm{e}$ & $7.9 \mathrm{~g}$ & $7.5 \mathrm{~h}$ & $7.8 \mathrm{~d}$ & $7.9 \mathrm{~g}$ & $8.0 \mathrm{f}$ & $8.5 \mathrm{~h}$ & $8.7 \mathrm{f}$ & $8.6 \mathrm{~h}$ & $8.2 \mathrm{~cd}$ \\
\hline 9-Rekafa & $8.0 \mathrm{~h}$ & $8.2 \mathrm{f}$ & $8.5 \mathrm{j}$ & $7.7 \mathrm{~h}$ & $6.8 \mathrm{~L}$ & $7.7 \mathrm{~g}$ & $7.3 \mathrm{~h}$ & $8.2 \mathrm{~d}$ & $7.8 \mathrm{~g}$ & $8.1 \mathrm{j}$ & $8.4 \mathrm{~h}$ & $8.8 \mathrm{f}$ & $7.9 \mathrm{de}$ \\
\hline 10-Jeous Q & $8.5 \mathrm{f}$ & $7.9 \mathrm{~g}$ & $8.9 \mathrm{~g}$ & $7.5 \mathrm{i}$ & $7.3 \mathrm{i}$ & $7.0 \mathrm{k}$ & $6.9 \mathrm{j}$ & $7.8 \mathrm{~h}$ & $8.2 \mathrm{e}$ & $8.3 \mathrm{i}$ & $8.7 \mathrm{f}$ & $8.9 \mathrm{e}$ & $7.9 \mathrm{de}$ \\
\hline 11-Muhand & $7.8 \mathrm{i}$ & $7.6 \mathrm{j}$ & $8.3 \mathrm{~L}$ & $7.9 \mathrm{f}$ & $6.6 n$ & $6.8 \mathrm{i}$ & $7.2 \mathrm{i}$ & $7.5 \mathrm{j}$ & $7.8 \mathrm{~g}$ & $8.0 \mathrm{k}$ & $8.5 \mathrm{~g}$ & $8.6 \mathrm{~h}$ & 7.7 ef \\
\hline 12-Farok Br. & $7.5 \mathrm{j}$ & $7.9 \mathrm{~g}$ & $8.5 \mathrm{j}$ & $7.4 j$ & $6.9 \mathrm{k}$ & $7.2 \mathrm{i}$ & $7.5 \mathrm{f}$ & $8.0 \mathrm{f}$ & $7.6 \mathrm{i}$ & $7.9 \mathrm{~L}$ & $8.1 \mathrm{j}$ & $8.8 \mathrm{f}$ & $7.7 \mathrm{ef}$ \\
\hline 13-Khane Br. & $7.2 \mathrm{~L}$ & $7.8 \mathrm{~h}$ & $8.7 \mathrm{~h}$ & $6.9 \mathrm{~L}$ & $6.7 \mathrm{~m}$ & $6.7 \mathrm{n}$ & $6.8 \mathrm{k}$ & $7.6 \mathrm{i}$ & $7.4 j$ & $8.1 \mathrm{j}$ & $8.3 \mathrm{i}$ & $8.5 \mathrm{i}$ & $7.5 \mathrm{fg}$ \\
\hline 14-Ashe jam & $7.4 \mathrm{k}$ & $7.7 \mathrm{i}$ & $8.4 \mathrm{k}$ & $7.2 \mathrm{k}$ & $6.8 \mathrm{~L}$ & $7.1 \mathrm{j}$ & $6.9 \mathrm{j}$ & $6.7 \mathrm{k}$ & $7.7 \mathrm{~h}$ & $7.8 \mathrm{~m}$ & $8.1 \mathrm{j}$ & $8.7 \mathrm{~g}$ & $7.5 \mathrm{fg}$ \\
\hline 15-Bedara & $7.1 \mathrm{~m}$ & $7.1 \mathrm{k}$ & $8.1 \mathrm{~m}$ & $6.7 \mathrm{~m}$ & $7.2 \mathrm{j}$ & $6.9 \mathrm{~L}$ & $7.4 \mathrm{~g}$ & $6.6 \mathrm{~L}$ & $7.4 \mathrm{j}$ & $6.9 n$ & $8.4 \mathrm{~h}$ & $8.5 \mathrm{i}$ & $7.3 \mathrm{~g}$ \\
\hline 16-Ibrhem Br. & $7.8 \mathrm{i}$ & $8.2 \mathrm{f}$ & $8.6 \mathrm{i}$ & $7.8 \mathrm{~g}$ & $8.0 \mathrm{f}$ & $7.0 \mathrm{k}$ & $8.1 \mathrm{a}$ & $7.9 \mathrm{~g}$ & $7.8 \mathrm{~g}$ & $8.1 \mathrm{j}$ & $8.8 \mathrm{e}$ & $8.9 \mathrm{e}$ & $8.0 \mathrm{~cd}$ \\
\hline
\end{tabular}

(BOD5) Biochemical Oxygen Demand in $\mathrm{mg} / \mathrm{L}$. (BOD5) is an important parameter of water quality for it greatly affects the value of DO in the water body. In this work the mean value of (BOD) and standard error of mean values were ranged from $(2.558 \pm 0.9317)$ to $(16.642 \pm 2.9525) \mathrm{mg} / \mathrm{L}$. (Table 15).The total mean value of (BOD) were increased the permissible limit. The permissible limit for BOD5 as per (WHO , 2011) is $5 \mathrm{mg} / \mathrm{L}$. During the study area, Table 6, the lowest value of BOD was recorded in site Jalke in February in the winter season was $1.6 \mathrm{mg} / \mathrm{L}$.The highest value was recorded in site Khane Bridge in August in the summer season was $19.9 \mathrm{mg} / \mathrm{L}$ .The high concentration of biochemical oxygen demand may be due to extensive uses of organic matter. The water samples from the sites Jalke, Btofa, Jamsemo, Aelol, Dalal, and jean site from the study area have BOD values less than the permissible limits. While the other samples were above the permissible limits. Increase in biochemical oxygen demand which is a reflection of bacterial oxygen demand leads to a decrease in DO and leads to hypoxia conditions. The studied River water has significant variation in (BOD5), at the study times. They have a significant difference at $\mathrm{p} \leq 0.05$ ) (Table 15). This work are significantly minimum than those obtained by (Bilyaminu., 2020)

Table 6 Monthly variation of (BOD) Biochemical oxygen demand $\mathrm{mg} / \mathrm{L}$, in sixteen locations

\begin{tabular}{|l|l|l|l|l|l|l|l|l|l|l|l|l|l|}
\hline $\begin{array}{l}\text { Months } \\
\text { Sites }\end{array}$ & Mar. & Apr. & May. & Jun. & Jul. & Aug. & Sept. & Oct. & Nov. & Dec. & Jan. & Feb & Average \\
\hline 1-Jalke & $3.2 \mathrm{~m}$ & $2.6 \mathrm{o}$ & $1.9 \mathrm{p}$ & $3.2 \mathrm{o}$ & $2.8 \mathrm{p}$ & $3.1 \mathrm{o}$ & $1.7 \mathrm{o}$ & $2.1 \mathrm{n}$ & $1.7 \mathrm{o}$ & $2.0 \mathrm{p}$ & $4.8 \mathrm{~L}$ & $1.6 \mathrm{o}$ & $2.8 \mathrm{i}$ \\
\hline 2-Batefa & $3.7 \mathrm{k}$ & $2.9 \mathrm{n}$ & $4.1 \mathrm{~L}$ & $3.1 \mathrm{p}$ & $3.5 \mathrm{n}$ & $2.8 \mathrm{p}$ & $2.1 \mathrm{n}$ & $1.8 \mathrm{p}$ & $1.9 \mathrm{n}$ & $2.6 \mathrm{o}$ & $3.5 \mathrm{n}$ & $4.0 \mathrm{~m}$ & $3.0 \mathrm{hi}$ \\
\hline 3-Jamsemo & $4.1 \mathrm{j}$ & $3.5 \mathrm{~L}$ & $4.0 \mathrm{~m}$ & $4.5 \mathrm{~m}$ & $2.9 \mathrm{o}$ & $3.6 \mathrm{n}$ & $2.8 \mathrm{~L}$ & $2.0 \mathrm{o}$ & $1.6 \mathrm{p}$ & $3.6 \mathrm{n}$ & $4.9 \mathrm{k}$ & $3.8 \mathrm{n}$ & $3.4 \mathrm{ghi}$ \\
\hline 4-Aelol Q & $3.6 \mathrm{~L}$ & $3.4 \mathrm{~m}$ & $2.5 \mathrm{o}$ & $5.0 \mathrm{k}$ & $3.6 \mathrm{~m}$ & $4.9 \mathrm{k}$ & $2.4 \mathrm{~m}$ & $2.7 \mathrm{~m}$ & $2.0 \mathrm{~m}$ & $4.7 \mathrm{k}$ & $4.3 \mathrm{~m}$ & $4.7 \mathrm{k}$ & $3.6 \mathrm{gh}$ \\
\hline 5-Dalal 1 & $3.2 \mathrm{~m}$ & $3.7 \mathrm{k}$ & $2.8 \mathrm{n}$ & $4.3 \mathrm{n}$ & $3.9 \mathrm{~L}$ & $4.4 \mathrm{~m}$ & $3.3 \mathrm{k}$ & $2.9 \mathrm{~L}$ & $2.6 \mathrm{~L}$ & $3.9 \mathrm{~m}$ & $5.0 \mathrm{j}$ & $4.6 \mathrm{~L}$ & $3.7 \mathrm{gh}$ \\
\hline 6-Dalal 2 & $4.1 \mathrm{j}$ & $5.0 \mathrm{j}$ & $4.9 \mathrm{k}$ & $4.7 \mathrm{~L}$ & $4.4 \mathrm{k}$ & $4.8 \mathrm{~L}$ & $3.7 \mathrm{j}$ & $4.2 \mathrm{j}$ & $3.6 \mathrm{j}$ & $4.2 \mathrm{~L}$ & $4.3 \mathrm{~m}$ & $4.8 \mathrm{j}$ & $4.3 \mathrm{~g}$ \\
\hline 7-Jean & $4.8 \mathrm{i}$ & $7.3 \mathrm{~h}$ & $5.5 \mathrm{j}$ & $8.1 \mathrm{i}$ & $5.9 \mathrm{j}$ & $7.7 \mathrm{j}$ & $4.2 \mathrm{i}$ & $3.9 \mathrm{k}$ & $3.5 \mathrm{k}$ & $5.8 \mathrm{j}$ & $5.2 \mathrm{i}$ & $5.8 \mathrm{i}$ & $5.6 \mathrm{f}$ \\
\hline 8-Hasanke & $6.2 \mathrm{~h}$ & $8.2 \mathrm{~g}$ & $7.4 \mathrm{i}$ & $6.8 \mathrm{j}$ & $14.6 \mathrm{f}$ & $12.5 \mathrm{i}$ & $9.4 \mathrm{~h}$ & $5.7 \mathrm{i}$ & $7.4 \mathrm{i}$ & $9.9 \mathrm{i}$ & $11 . \mathrm{h} 3$ & $9.9 \mathrm{~h}$ & $9.1 \mathrm{e}$ \\
\hline 9-Rekafa & $8.6 \mathrm{~g}$ & $6.9 \mathrm{i}$ & $8.3 \mathrm{~h}$ & $9.4 \mathrm{~h}$ & $12.9 \mathrm{~h}$ & $12.8 \mathrm{~h}$ & $16.9 \mathrm{~d}$ & $11.5 \mathrm{~h}$ & $13.5 \mathrm{~g}$ & $19.5 \mathrm{a}$ & $13.7 \mathrm{e}$ & $14.7 \mathrm{e}$ & $13.4 \mathrm{~d}$ \\
\hline 10-Jeous Q & $10.7 \mathrm{e}$ & $14.1 \mathrm{~d}$ & $11.7 \mathrm{f}$ & $19.3 \mathrm{a}$ & $10.2 \mathrm{i}$ & $13.6 \mathrm{~g}$ & $17.3 \mathrm{c}$ & $14.9 \mathrm{~d}$ & $16.9 \mathrm{c}$ & $16.6 \mathrm{f}$ & $17.4 \mathrm{~b}$ & $19.5 \mathrm{a}$ & $14.3 \mathrm{~b}$ \\
\hline 11-Muhand & $12.8 \mathrm{~d}$ & $15.4 \mathrm{c}$ & $15.5 \mathrm{e}$ & $14.2 \mathrm{f}$ & $13.6 \mathrm{~g}$ & $15.1 \mathrm{e}$ & $19.5 \mathrm{a}$ & $15.0 \mathrm{c}$ & $17.1 \mathrm{~b}$ & $17.9 \mathrm{~d}$ & $14.1 \mathrm{~d}$ & $17.6 \mathrm{~b}$ & $15.6 \mathrm{ab}$ \\
\hline 12-Farok B & $14.6 \mathrm{c}$ & $16.9 \mathrm{~b}$ & $19.3 \mathrm{a}$ & $16.0 \mathrm{~d}$ & $15.1 \mathrm{e}$ & $22.7 \mathrm{a}$ & $18.1 \mathrm{~b}$ & $14.6 \mathrm{f}$ & $14.0 \mathrm{f}$ & $17.1 \mathrm{e}$ & $11.8 \mathrm{~g}$ & $19.5 \mathrm{a}$ & $16.6 \mathrm{a}$ \\
\hline
\end{tabular}




\begin{tabular}{|l|l|l|l|l|l|l|l|l|l|l|l|l|l|}
\hline 13-Khane B & $12.8 \mathrm{~d}$ & $14.1 \mathrm{~d}$ & $18.2 \mathrm{c}$ & $14.7 \mathrm{e}$ & $17.3 \mathrm{~b}$ & $19.9 \mathrm{c}$ & $15.0 \mathrm{e}$ & $19.1 \mathrm{a}$ & $15.8 \mathrm{~d}$ & $18.0 \mathrm{c}$ & $19.5 \mathrm{a}$ & $11.9 \mathrm{~g}$ & $16.3 \mathrm{a}$ \\
\hline 14-Ashe jam & $16,9 \mathrm{a}$ & $19.3 \mathrm{a}$ & $17.1 \mathrm{~d}$ & $16.6 \mathrm{~b}$ & $17.2 \mathrm{c}$ & $18.6 \mathrm{~d}$ & $12.3 \mathrm{~g}$ & $15.8 \mathrm{~b}$ & $17.8 \mathrm{a}$ & $14.1 \mathrm{~h}$ & $15.2 \mathrm{c}$ & $15.8 \mathrm{~d}$ & $16.4 \mathrm{a}$ \\
\hline 15-Bedara & $15.9 \mathrm{~b}$ & $12.6 \mathrm{e}$ & $18.5 \mathrm{~b}$ & $16.2 \mathrm{c}$ & $19.0 \mathrm{a}$ & $13.8 \mathrm{f}$ & $14.6 \mathrm{f}$ & $13.0 \mathrm{~g}$ & $15.0 \mathrm{e}$ & $19.4 \mathrm{~b}$ & $14.1 \mathrm{~d}$ & $17.1 \mathrm{c}$ & $15.7 \mathrm{ab}$ \\
\hline 16-Ibrhem B & $9,5 \mathrm{f}$ & $11.6 \mathrm{f}$ & $10.3 \mathrm{~g}$ & $13.7 \mathrm{~g}$ & $16.9 \mathrm{~d}$ & $21.0 \mathrm{~b}$ & $16.9 \mathrm{~d}$ & $14.7 \mathrm{e}$ & $9.6 \mathrm{~h}$ & $15.3 \mathrm{~g}$ & $13.6 \mathrm{f}$ & $12.8 \mathrm{f}$ & $13.8 \mathrm{c}$ \\
\hline
\end{tabular}

Total hardness as $\mathrm{CaCO} 3(\mathrm{TH})$ in $(\mathrm{mg} / \mathrm{L})$ : The $(\mathrm{TH})$ of the River water is dependent on the presence of $\left(\mathrm{Mg}^{2+}\right)\left(\mathrm{Ca}^{2+}\right)$ and $\mathrm{HCO}^{-}$- ions in the water which cause the hard water. In this study the mean value of $(\mathrm{TH})$ and standard error of mean values was $(434.58 \pm 11.843)$ to $(636.08 \pm 54.199) \mathrm{mg} / \mathrm{L}$. (Table 15), The mean value of $(\mathrm{TH})$ were exceeded the permissible limit .The safe level of drinking water for hardness is $300 \mathrm{mg} / \mathrm{L}$, set by (WHO ., 2012). In this work the concentrations of (TH) at all sampling sites (Table 7), it ranged from (419 to 687) $\mathrm{mg} / \mathrm{L}$ .The highest concentration was observed at site Bedara in July.

\begin{tabular}{|c|c|c|c|c|c|c|c|c|c|c|c|c|c|}
\hline $\begin{array}{l}\text { Months } \\
\text { Sites }\end{array}$ & Mar. & Apr. & May. & Jun. & Jul. & Aug. & Sept. & Oct. & Nov. & Dec. & Jan. & Feb. & $\begin{array}{l}\text { Avera } \\
\text { ge }\end{array}$ \\
\hline 1-Jalke & $427 \mathrm{o}$ & $432 \mathrm{o}$ & $419 n$ & $426 p$ & $434 \mathrm{o}$ & 4410 & 4280 & 4330 & $427 n$ & $436 n$ & 449 o & 463 o & $434 \mathrm{k}$ \\
\hline 2-Batefa & $431 \mathrm{n}$ & $430 \mathrm{p}$ & $425 \mathrm{~m}$ & $429 n$ & $421 p$ & $438 p$ & $431 n$ & $431 \mathrm{p}$ & 4250 & $439 \mathrm{~L}$ & $446 \mathrm{p}$ & $471 \mathrm{~m}$ & $436 \mathrm{k}$ \\
\hline 3-Jamsmo & $444 \mathrm{~m}$ & $451 \mathrm{n}$ & $431 \mathrm{~L}$ & $427 \mathrm{o}$ & $436 n$ & $442 n$ & $452 \mathrm{~m}$ & $442 n$ & $431 \mathrm{~L}$ & $437 \mathrm{~m}$ & $451 n$ & $469 n$ & $449 \mathrm{k}$ \\
\hline 4-Aelol Q & $540 \mathrm{~L}$ & $539 \mathrm{~m}$ & $497 \mathrm{k}$ & $469 \mathrm{~L}$ & $444 m$ & $449 m$ & $459 \mathrm{~L}$ & $451 \mathrm{~m}$ & $429 m$ & $435 \mathrm{o}$ & $485 \mathrm{~L}$ & $491 \mathrm{k}$ & $474 j$ \\
\hline 5-Dalal Br. & $553 \mathrm{k}$ & $561 \mathrm{~L}$ & $518 \mathrm{j}$ & $449 m$ & $451 \mathrm{~L}$ & $482 \mathrm{~L}$ & $478 \mathrm{j}$ & $466 \mathrm{~L}$ & $441 \mathrm{k}$ & $449 \mathrm{k}$ & $462 m$ & $487 \mathrm{~L}$ & $483 \mathrm{ij}$ \\
\hline 6-Solaf & $568 \mathrm{j}$ & $589 \mathrm{k}$ & $563 \mathrm{i}$ & $471 \mathrm{k}$ & $466 \mathrm{~h}$ & $487 \mathrm{k}$ & $471 \mathrm{k}$ & $483 j$ & $453 \mathrm{j}$ & $461 \mathrm{i}$ & $489 \mathrm{k}$ & $519 \mathrm{j}$ & $497 \mathrm{hi}$ \\
\hline 7-Jean & $586 \mathrm{i}$ & $621 \mathrm{i}$ & $570 \mathrm{~h}$ & $483 j$ & $497 \mathrm{j}$ & $505 \mathrm{j}$ & $497 \mathrm{i}$ & $478 \mathrm{k}$ & $461 \mathrm{i}$ & $459 \mathrm{j}$ & $511 \mathrm{j}$ & $538 \mathrm{i}$ & $557 \mathrm{gh}$ \\
\hline 8-Hasanke & $613 \mathrm{~g}$ & $634 \mathrm{~g}$ & $594 d$ & $499 \mathrm{i}$ & $524 \mathrm{i}$ & $531 \mathrm{i}$ & $511 \mathrm{~h}$ & $488 \mathrm{i}$ & $475 \mathrm{~h}$ & $466 \mathrm{~h}$ & $533 \mathrm{i}$ & $544 \mathrm{~h}$ & $534 \mathrm{fg}$ \\
\hline 9-Rekafa & $643 \mathrm{e}$ & $649 \mathrm{~d}$ & $612 \mathrm{c}$ & $589 \mathrm{~g}$ & $599 \mathrm{~g}$ & $578 \mathrm{~g}$ & $529 \mathrm{~g}$ & $491 \mathrm{~h}$ & $499 \mathrm{~g}$ & $513 \mathrm{~g}$ & $546 \mathrm{~g}$ & $563 \mathrm{~g}$ & 567 e \\
\hline 10- Jeous Q & $613 \mathrm{~g}$ & $627 \mathrm{~h}$ & $584 \mathrm{~g}$ & $595 \mathrm{f}$ & $614 f$ & $611 \mathrm{f}$ & $551 \mathrm{f}$ & $525 \mathrm{~g}$ & $511 \mathrm{f}$ & $526 \mathrm{e}$ & $552 \mathrm{f}$ & $574 \mathrm{f}$ & $573 \mathrm{de}$ \\
\hline 11-Muhand & $688 \mathrm{~b}$ & $671 \mathrm{c}$ & $592 \mathrm{e}$ & $610 \mathrm{e}$ & $621 \mathrm{e}$ & $623 \mathrm{e}$ & $583 \mathrm{~d}$ & $537 \mathrm{f}$ & $531 \mathrm{e}$ & $519 \mathrm{f}$ & $563 \mathrm{e}$ & $579 \mathrm{e}$ & $591 \mathrm{~cd}$ \\
\hline 12-Farok Br. & $642 \mathrm{f}$ & $639 \mathrm{f}$ & $570 \mathrm{~h}$ & $633 \mathrm{~d}$ & $642 d$ & $637 d$ & $579 \mathrm{e}$ & $541 \mathrm{e}$ & $552 \mathrm{c}$ & $537 \mathrm{c}$ & $578 \mathrm{~d}$ & $583 \mathrm{~d}$ & $594 \mathrm{~cd}$ \\
\hline 13-Khane Br. & $661 \mathrm{c}$ & $673 \mathrm{~b}$ & $612 \mathrm{c}$ & $639 \mathrm{c}$ & $649 \mathrm{c}$ & $651 \mathrm{c}$ & $593 \mathrm{a}$ & $544 \mathrm{c}$ & $539 \mathrm{~d}$ & $539 \mathrm{~b}$ & $591 \mathrm{c}$ & $611 \mathrm{c}$ & $608 \mathrm{bc}$ \\
\hline 14 Ashe jam & $659 \mathrm{~d}$ & $648 \mathrm{e}$ & $631 \mathrm{~b}$ & $641 \mathrm{~b}$ & $674 b$ & $659 \mathrm{~b}$ & $584 \mathrm{c}$ & $563 \mathrm{~b}$ & $561 \mathrm{~b}$ & $542 \mathrm{a}$ & $613 \mathrm{~b}$ & $638 \mathrm{~b}$ & $619 \mathrm{ab}$ \\
\hline 15 Bedara & $692 \mathrm{a}$ & $701 \mathrm{a}$ & $674 a$ & $663 a$ & $687 \mathrm{a}$ & $661 \mathrm{a}$ & $591 b$ & $573 \mathrm{a}$ & $586 \mathrm{a}$ & $531 \mathrm{~d}$ & $633 \mathrm{a}$ & $641 \mathrm{a}$ & $636 \mathrm{a}$ \\
\hline 16-Ibrahem Br. & $596 \mathrm{~h}$ & $611 \mathrm{j}$ & $589 \mathrm{f}$ & $562 \mathrm{~h}$ & $577 \mathrm{~h}$ & $569 \mathrm{~h}$ & $551 \mathrm{f}$ & $543 \mathrm{~d}$ & $427 n$ & $431 p$ & $544 \mathrm{~h}$ & $563 \mathrm{~g}$ & $547 \mathrm{f}$ \\
\hline
\end{tabular}

Calcium $\left(\mathrm{Ca}^{2+}\right)$ : in this work the mean value of $\left(\mathrm{Ca}^{2+}\right)$ and standard error of mean values were ranged from (321.00 \pm $36.636)$ to $(636.08 \pm 54.199) \mathrm{mg} / \mathrm{L}$. (Table 15$)$, The mean value of $\left(\mathrm{Ca}^{2+)}\right.$ were exceeded the permissible limit, The safe level of $\left(\mathrm{Ca}^{2+}\right)$ value for domestic purpose is $75 \mathrm{mg} / \mathrm{L}$ (WHO, 2011).(Table 8). The value of $\left(\mathrm{Ca}^{2+}\right.$ ranged between (278 to 493$) \mathrm{mg} / \mathrm{L}$, the highest value of calcium was recorded at site Bedara in February. The rapid urbanization and
The high value of total hardness of samples may be due to geological formation, while the minimum concentration was observed at site Jalke in May was $419 \mathrm{mg} / \mathrm{L}$. The concentration of total hardness at all sites were exceeded the safe level (300 $\mathrm{mg} / \mathrm{L}$ ). And mostly exceeds the maximum permissible limits $(500 \mathrm{mg} / \mathrm{L})$. (WHO, 2011) .The studied River water has significant variation in $(\mathrm{TH})$ at the study times .They have a significant difference at $p \leq 0.05$ ) (Table 15). In this work are significantly maximum than those obtained by ( Saurabh et al., 2021).

ness (TH) $\mathrm{mg} / \mathrm{L}$ in sixteen locations

industrialization in the area lead to the high value of calcium. While the minimum value was observed at site Batefa was 278 $\mathrm{mg} / \mathrm{L}$ in September. All the samples exceeded the permissible limit. The studied River water exhibit significant variation in $\left(\left(\mathrm{Ca}^{2+}\right)\right.$ in the study area . They have a significant variation at ( $p \leq 0.05$ ) (Table 15). In this work are significantly higher than those obtained by (NidhiGupta et al., 2017).

Table 8 Monthly variation of Calcium hardness $\left(\mathrm{Ca}^{2+}\right) \mathrm{in} \mathrm{mg} / \mathrm{L}$ in sixteen locations

\begin{tabular}{|c|c|c|c|c|c|c|c|c|c|c|c|c|c|}
\hline $\begin{array}{l}\text { Months } \\
\text { Sites }\end{array}$ & Mar. & Apr. & May. & Jun. & Jul. & Aug. & Sept. & Oct. & Nov. & Dec. & Jan. & Feb. & Average \\
\hline 1-Jalke & $318 n$ & $321 \mathrm{n}$ & $338 \mathrm{~L}$ & $298 \mathrm{~m}$ & $331 \mathrm{~m}$ & $346 \mathrm{o}$ & $361 \mathrm{~m}$ & $382 \mathrm{~m}$ & $341 \mathrm{o}$ & $393 m$ & $386 \mathrm{~L}$ & $391 \mathrm{~L}$ & $350 \mathrm{~g}$ \\
\hline 2-Batefa & 2950 & $341 \mathrm{~L}$ & 2950 & $337 \mathrm{~L}$ & $371 \mathrm{~L}$ & $286 \mathrm{p}$ & $278 n$ & $362 n$ & $355 \mathrm{~m}$ & 2910 & 2790 & $362 n$ & $290 \mathrm{~h}$ \\
\hline 3-Jamsemo & $352 \mathrm{~L}$ & $361 \mathrm{k}$ & $337 \mathrm{~m}$ & $351 \mathrm{k}$ & $385 \mathrm{k}$ & $391 \mathrm{k}$ & $375 \mathrm{~L}$ & 3580 & $349 n$ & $363 n$ & $361 n$ & $357 \mathrm{o}$ & $361 \mathrm{f}$ \\
\hline 4-Aelol Q & $327 \mathrm{~m}$ & $340 \mathrm{~m}$ & $339 \mathrm{k}$ & $363 \mathrm{i}$ & $371 \mathrm{~L}$ & $382 \mathrm{~m}$ & $379 \mathrm{k}$ & $383 \mathrm{~L}$ & $369 \mathrm{~L}$ & $394 \mathrm{~L}$ & $385 \mathrm{~m}$ & $391 \mathrm{~L}$ & $368 \mathrm{f}$ \\
\hline 5-Dalal 1 & $386 \mathrm{k}$ & 2910 & $327 n$ & $362 \mathrm{j}$ & $385 \mathrm{k}$ & $373 n$ & $388 \mathrm{j}$ & $396 \mathrm{k}$ & $411 \mathrm{j}$ & $407 \mathrm{k}$ & $421 \mathrm{i}$ & $418 \mathrm{k}$ & $378 \mathrm{e}$ \\
\hline 6-Dalal 2 & $395 \mathrm{j}$ & $417 \mathrm{f}$ & $396 \mathrm{j}$ & $386 \mathrm{~h}$ & $410 \mathrm{j}$ & $396 \mathrm{j}$ & $414 \mathrm{~h}$ & $227 p$ & $418 \mathrm{i}$ & $431 \mathrm{~h}$ & $411 \mathrm{j}$ & $427 \mathrm{j}$ & $410 \mathrm{~d}$ \\
\hline 7-Jean & $429 \mathrm{~d}$ & $399 \mathrm{i}$ & $418 \mathrm{~g}$ & $426 f$ & $419 \mathrm{i}$ & $425 \mathrm{~g}$ & $439 \mathrm{c}$ & $426 \mathrm{i}$ & $419 \mathrm{~h}$ & $443 \mathrm{e}$ & $421 \mathrm{i}$ & $430 \mathrm{i}$ & $424 \mathrm{c}$ \\
\hline 8-Hasanke & $417 \mathrm{e}$ & $426 \mathrm{~d}$ & $411 \mathrm{~h}$ & $430 \mathrm{~d}$ & $427 \mathrm{f}$ & $431 \mathrm{f}$ & $428 \mathrm{f}$ & $434 \mathrm{e}$ & $439 d$ & $451 \mathrm{c}$ & $447 \mathrm{f}$ & $445 \mathrm{f}$ & $432 \mathrm{c}$ \\
\hline 9-Rekafa & $405 \mathrm{~h}$ & $395 \mathrm{j}$ & $436 \mathrm{~d}$ & $417 \mathrm{~g}$ & $426 \mathrm{~g}$ & $419 \mathrm{i}$ & $433 \mathrm{e}$ & $425 \mathrm{j}$ & $437 \mathrm{e}$ & $441 \mathrm{f}$ & $428 \mathrm{~h}$ & $451 d$ & $426 \mathrm{c}$ \\
\hline 10-Jeous Q & $399 \mathrm{i}$ & $410 \mathrm{~h}$ & $422 \mathrm{f}$ & $426 \mathrm{f}$ & $431 \mathrm{e}$ & $424 \mathrm{~h}$ & $428 \mathrm{f}$ & $431 \mathrm{f}$ & $420 \mathrm{~g}$ & $436 \mathrm{~g}$ & $441 \mathrm{~g}$ & $434 \mathrm{~h}$ & $425 \mathrm{c}$ \\
\hline 11-Muhand & $416 f$ & $421 \mathrm{e}$ & $427 \mathrm{e}$ & $429 \mathrm{e}$ & $441 d$ & $438 \mathrm{e}$ & $426 \mathrm{~g}$ & $428 \mathrm{~g}$ & $431 \mathrm{f}$ & $427 \mathrm{i}$ & $455 \mathrm{~d}$ & $441 \mathrm{~g}$ & $431 \mathrm{c}$ \\
\hline 12-Farok Br & $461 \mathrm{~b}$ & $436 c$ & $445 c$ & $436 c$ & $455 c$ & $461 \mathrm{c}$ & $439 \mathrm{c}$ & $447 \mathrm{~d}$ & $451 \mathrm{c}$ & $449 d$ & $462 \mathrm{c}$ & $473 c$ & $451 \mathrm{~b}$ \\
\hline 13-Khane $\mathrm{Br}$ & $467 \mathrm{a}$ & $459 \mathrm{~b}$ & $451 \mathrm{~b}$ & $464 a$ & $458 \mathrm{~b}$ & $476 a$ & $481 \mathrm{a}$ & $479 a$ & $483 a$ & $471 b$ & $484 a$ & $491 \mathrm{~b}$ & $472 \mathrm{a}$ \\
\hline 14-Ashe jam & $386 \mathrm{k}$ & $411 \mathrm{~g}$ & $396 \mathrm{j}$ & $430 \mathrm{~d}$ & $427 \mathrm{f}$ & $448 \mathrm{~d}$ & $435 \mathrm{~d}$ & $450 \mathrm{c}$ & $439 \mathrm{~d}$ & $427 \mathrm{i}$ & $451 \mathrm{e}$ & $448 \mathrm{e}$ & $429 \mathrm{c}$ \\
\hline 15-Bedara & $449 \mathrm{c}$ & $463 a$ & $459 a$ & $462 b$ & $473 a$ & $469 \mathrm{~b}$ & $471 b$ & $466 \mathrm{~b}$ & $482 b$ & $473 a$ & $477 b$ & $493 a$ & $469 \mathrm{a}$ \\
\hline 16-Ibrhem Br & $413 \mathrm{~g}$ & $395 \mathrm{j}$ & $410 \mathrm{i}$ & $386 \mathrm{~h}$ & $425 \mathrm{~h}$ & $385 \mathrm{~L}$ & $411 \mathrm{i}$ & $427 \mathrm{~h}$ & $379 \mathrm{k}$ & $410 \mathrm{j}$ & $395 \mathrm{k}$ & $376 \mathrm{~m}$ & $401 \mathrm{~d}$ \\
\hline
\end{tabular}


Magnesium $\left(\mathrm{Mg}^{2+}\right)$ in $\mathrm{mg} / \mathrm{L}$ : In this study the mean value of $\left(\mathrm{Mg}^{2+}\right)$ and standard error of mean values were $(80.25 \pm$ $15.915)$ to $(188.75 \pm 55.464) \mathrm{mg} / \mathrm{L}$. (Table 15$)$, The mean value of $\left(\mathrm{Mg}^{2+}\right)$.were exceeded the safe level, The acceptable limit of $\left(\mathrm{Mg}^{2+}\right)$. According to (WHO , 2012) is $30 \mathrm{mg} / \mathrm{l}$ and permissible limit is $100 \mathrm{mg} / \mathrm{l}$ (Table 9). The range of magnesium in River water samples was (30 to273 ) $\mathrm{mg} / \mathrm{l}$ the highest concentration was observed at location ashejame and the lowest value was in site Dalal . (Table15) shows a significant variation in the $\left(\mathrm{Mg}^{2+}\right)$ among the studied River water. They have a significant difference at $(p \leq 0.05)$. In this work are significantly higher than those obtained by (ackson et al ., 2020

Table 9 Monthly variation of Magnesium hardness $\left(\mathrm{Mg}^{2+}\right)$ in $\mathrm{mg} / \mathrm{l}$ in sixteen locations

\begin{tabular}{|c|c|c|c|c|c|c|c|c|c|c|c|c|c|}
\hline $\begin{array}{ll}\text { Months } \\
\text { Sites }\end{array}$ & Mar. & Apr. & May. & Jun. & Jul. & Aug. & Sept. & Oct. & Nov. & Dec. & Jan. & Feb. & Average \\
\hline 1-Jalke & $109 \mathrm{o}$ & $111 \mathrm{n}$ & $81 \mathrm{p}$ & $128 \mathrm{j}$ & $103 \mathrm{i}$ & $95 \mathrm{k}$ & $67 \mathrm{~m}$ & 510 & $86 \mathrm{f}$ & $43 \mathrm{j}$ & $63 n$ & $72 \mathrm{o}$ & $84 \mathrm{~h}$ \\
\hline 2-Batefa & $138 \mathrm{n}$ & $89 p$ & $130 \mathrm{~m}$ & $92 \mathrm{~L}$ & $50 \mathrm{o}$ & $152 \mathrm{~h}$ & $153 b$ & $69 \mathrm{~h}$ & $70 \mathrm{~h}$ & $148 \mathrm{a}$ & $167 \mathrm{a}$ & $159 \mathrm{c}$ & $144 \mathrm{def}$ \\
\hline 3-Jamsemo & $92 \mathrm{p}$ & $90 \mathrm{o}$ & $94 \mathrm{o}$ & 760 & $51 n$ & 510 & $77 \mathrm{~L}$ & $84 \mathrm{f}$ & $82 \mathrm{~g}$ & $74 \mathrm{f}$ & $90 \mathrm{k}$ & $102 \mathrm{k}$ & $81 \mathrm{~h}$ \\
\hline 4-Aelol Q & $213 \mathrm{f}$ & $199 \mathrm{~L}$ & $158 \mathrm{k}$ & $106 \mathrm{k}$ & $73 \mathrm{~L}$ & $76 n$ & $80 \mathrm{k}$ & $68 \mathrm{i}$ & $60 \mathrm{j}$ & $41 \mathrm{~L}$ & $100 \mathrm{j}$ & $100 \mathrm{~L}$ & $106 \mathrm{fgh}$ \\
\hline 5-Dalal 1 & $167 \mathrm{k}$ & $270 a$ & $191 \mathrm{c}$ & $87 \mathrm{~m}$ & $66 \mathrm{~m}$ & $109 \mathrm{i}$ & $90 \mathrm{i}$ & $70 \mathrm{~g}$ & $30 p$ & $42 \mathrm{k}$ & $41 \mathrm{o}$ & $69 p$ & $111 \mathrm{fgh}$ \\
\hline 6-Dalal 2 & $153 \mathrm{~m}$ & $172 \mathrm{~m}$ & $167 \mathrm{~g}$ & $85 n$ & $36 p$ & $91 \mathrm{~L}$ & $57 \mathrm{o}$ & $56 \mathrm{~L}$ & 350 & $30 \mathrm{~m}$ & $78 \mathrm{~m}$ & $92 n$ & $87 \mathrm{gh}$ \\
\hline 7-Jean & $157 \mathrm{~L}$ & $222 \mathrm{f}$ & $152 \mathrm{~L}$ & $57 \mathrm{p}$ & $78 \mathrm{k}$ & $86 \mathrm{~m}$ & $58 n$ & $52 n$ & $42 \mathrm{~m}$ & 160 & $90 \mathrm{k}$ & $158 \mathrm{~d}$ & $133 \mathrm{fgh}$ \\
\hline 8-Hasanke & $196 \mathrm{~g}$ & $208 \mathrm{j}$ & $183 \mathrm{~d}$ & $190 \mathrm{~d}$ & $97 j$ & $100 \mathrm{j}$ & $83 \mathrm{j}$ & $54 \mathrm{~m}$ & $36 n$ & $15 p$ & $86 \mathrm{~L}$ & $99 \mathrm{~m}$ & $152 \mathrm{efg}$ \\
\hline 9-Rekafa & $238 \mathrm{~d}$ & $254 \mathrm{~b}$ & $176 \mathrm{f}$ & $172 \mathrm{~h}$ & $173 \mathrm{~h}$ & $109 \mathrm{i}$ & $96 \mathrm{~h}$ & $66 \mathrm{j}$ & $62 \mathrm{i}$ & $72 \mathrm{~g}$ & $118 \mathrm{e}$ & $112 \mathrm{i}$ & $144 \mathrm{cde}$ \\
\hline 10-Jeous Q & $214 \mathrm{e}$ & $217 \mathrm{~g}$ & $162 \mathrm{i}$ & $169 \mathrm{i}$ & $183 \mathrm{f}$ & $187 \mathrm{c}$ & $123 \mathrm{e}$ & $94 \mathrm{e}$ & $91 \mathrm{e}$ & $90 \mathrm{~d}$ & $111 \mathrm{~g}$ & $140 \mathrm{f}$ & $148 b c$ \\
\hline 11-Muhand & $272 \mathrm{~b}$ & $250 \mathrm{c}$ & $165 \mathrm{~h}$ & $181 \mathrm{e}$ & $180 \mathrm{~g}$ & $185 \mathrm{~d}$ & $157 \mathrm{a}$ & $109 \mathrm{c}$ & $100 \mathrm{~d}$ & $92 \mathrm{c}$ & $108 \mathrm{~h}$ & $138 \mathrm{~g}$ & $160 \mathrm{bc}$ \\
\hline 12-Farok Br. & $181 \mathrm{j}$ & $203 \mathrm{k}$ & $125 n$ & $197 \mathrm{c}$ & $187 \mathrm{e}$ & $176 \mathrm{f}$ & $140 \mathrm{~d}$ & $94 \mathrm{e}$ & $101 \mathrm{c}$ & $88 \mathrm{e}$ & $116 \mathrm{f}$ & $110 \mathrm{j}$ & $142 \mathrm{bcd}$ \\
\hline 13-Khane $\mathrm{Br}$. & $194 \mathrm{~h}$ & $214 \mathrm{i}$ & $161 \mathrm{j}$ & $175 \mathrm{~g}$ & 191d & $175 \mathrm{~g}$ & $112 \mathrm{~g}$ & $65 \mathrm{k}$ & $56 \mathrm{k}$ & $68 \mathrm{~h}$ & $107 \mathrm{i}$ & $120 \mathrm{~h}$ & $136 \mathrm{cde}$ \\
\hline 14-Ashe jam & $273 \mathrm{a}$ & $237 \mathrm{e}$ & $235 \mathrm{a}$ & $211 \mathrm{a}$ & $247 \mathrm{~b}$ & $210 \mathrm{a}$ & $149 \mathrm{c}$ & $113 \mathrm{~b}$ & $122 a$ & $115 \mathrm{~b}$ & $162 \mathrm{~b}$ & $191 \mathrm{a}$ & $190 \mathrm{a}$ \\
\hline 15-Bedara & $243 c$ & $238 \mathrm{~d}$ & $215 \mathrm{~b}$ & $201 \mathrm{~b}$ & $214 c$ & $192 b$ & $120 \mathrm{f}$ & $107 d$ & $104 \mathrm{~b}$ & $58 \mathrm{i}$ & $156 \mathrm{c}$ & $148 \mathrm{e}$ & $167 \mathrm{ab}$ \\
\hline 16-Ibrhem Br. & $183 \mathrm{i}$ & $216 \mathrm{~h}$ & $179 \mathrm{e}$ & $176 \mathrm{f}$ & $252 a$ & $184 \mathrm{e}$ & $140 \mathrm{~d}$ & $116 a$ & $48 \mathrm{~L}$ & $21 n$ & $149 \mathrm{~d}$ & $187 \mathrm{~b}$ & $146 b c$ \\
\hline
\end{tabular}

Chloride (CL-) : This work the mean value of (CL-) and standard error of mean values was ranged from $(21.825 \pm 2.2947)$ to $(39.300 \pm 47.3585) \mathrm{mg} / \mathrm{L}$. (Table 15$)$, The mean value of (CL-) obtained at Khabur River were within the safe concentration of chloride for safe drinking water according to (WHO.,2012) $(250 \mathrm{mg} / \mathrm{L})$. (Table 10). The highest value $(39.8 \mathrm{mg} / \mathrm{L})$ was recorded in Khane Bridge in the month of May
, The higher content of (CL-), in Khabur River may be due to animal origins like sewage inflow and human faces, and the lowest value $(18.3 \mathrm{mg} / \mathrm{L})$ in location Batofa at November. (Table 15), observes a significant difference in the (CL-) at the studied River water (Table 2). They have a significant difference at $(\mathrm{p} \leq 0.05)$. This work are significantly minimum than those obtained by (Musher ., 2015).

Table 10 Monthly variation of Chloride (CL-) in $\mathrm{mg} / \mathrm{l}$ in sixteen locations

\begin{tabular}{|c|c|c|c|c|c|c|c|c|c|c|c|c|c|}
\hline \begin{tabular}{ll}
\multicolumn{1}{l}{ Months } \\
Sites
\end{tabular} & Mar. & Apr & May. & Jun. & Jul. & Aug. & Sept. & Oct. & Nov. & Dec. & Jan & Feb & Average \\
\hline 1-Jalke & $19.7 \mathrm{k}$ & $20.1 \mathrm{~m}$ & $27.3 \mathrm{f}$ & $19.1 \mathrm{n}$ & $21.9 \mathrm{i}$ & $23.6 \mathrm{f}$ & $20.9 \mathrm{~h}$ & $21.5 \mathrm{~m}$ & $\begin{array}{l}21.0 \\
\mathrm{~L}\end{array}$ & $22.8 \mathrm{j}$ & $20.1 \mathrm{k}$ & $23.9 \mathrm{f}$ & $25.1 \mathrm{~h}$ \\
\hline 2-Batefa & 18,30 & $19.5 n$ & $22.1 \mathrm{j}$ & $18.6 \mathrm{o}$ & $20.8 \mathrm{k}$ & $22.4 \mathrm{~g}$ & $18.4 \mathrm{~m}$ & $21.6 \mathrm{~L}$ & $19.6 \mathrm{n}$ & $23.7 \mathrm{~h}$ & $28.1 \mathrm{~b}$ & $21.4 \mathrm{k}$ & $21.2 \mathrm{~h}$ \\
\hline 3-Jamsemo & $19.3 \mathrm{~L}$ & $21.4 \mathrm{~L}$ & $19.3 n$ & $22.6 \mathrm{i}$ & $25.1 \mathrm{f}$ & $19.4 \mathrm{k}$ & $18.8 \mathrm{~L}$ & $26.4 \mathrm{f}$ & $21.9 \mathrm{k}$ & $28.5 \mathrm{c}$ & $22.8 \mathrm{~g}$ & $26.0 \mathrm{e}$ & 22.6 fgh \\
\hline 4-Aelol Q & $19,1 \mathrm{~m}$ & $18.4 \mathrm{o}$ & $21.6 \mathrm{k}$ & $19.6 \mathrm{~L}$ & $27.3 \mathrm{~d}$ & $21.7 \mathrm{~h}$ & $19.6 \mathrm{j}$ & $22.7 \mathrm{k}$ & $24.1 \mathrm{j}$ & $23.2 \mathrm{i}$ & $25.0 \mathrm{e}$ & $22.6 \mathrm{~h}$ & 22.1gh \\
\hline 5-Dalal 1 & $18.4 n$ & $22.5 \mathrm{k}$ & $28.3 \mathrm{e}$ & $25.0 \mathrm{~g}$ & $22.4 \mathrm{~h}$ & $28.1 \mathrm{~d}$ & $24.2 \mathrm{f}$ & $30.0 \mathrm{e}$ & $26.7 \mathrm{~h}$ & $29.3 b$ & $21.1 \mathrm{j}$ & $18.5 \mathrm{o}$ & 26.6def \\
\hline 6-Dalal 2 & $23.9 \mathrm{~h}$ & $26.3 \mathrm{~g}$ & $31.0 \mathrm{~d}$ & $28.4 \mathrm{c}$ & $29.4 \mathrm{c}$ & $26.1 \mathrm{e}$ & $18.9 \mathrm{k}$ & $24.1 \mathrm{~h}$ & $31.0 \mathrm{~d}$ & $27.2 \mathrm{e}$ & $27.9 \mathrm{c}$ & $21.3 \mathrm{~L}$ & $25.5 \mathrm{~cd}$ \\
\hline 7-Jean & $29.5 \mathrm{f}$ & $31.3 \mathrm{c}$ & $25.2 \mathrm{~h}$ & $19.5 \mathrm{~m}$ & $18.8 \mathrm{~m}$ & $18.9 \mathrm{~L}$ & $18.4 \mathrm{~m}$ & $20.6 n$ & $27.5 \mathrm{~g}$ & $23.9 \mathrm{~g}$ & $21.6 \mathrm{i}$ & $20.5 n$ & $24.8 \mathrm{e}-\mathrm{h}$ \\
\hline 8-Hasanke & $27.0 \mathrm{~g}$ & $29.5 \mathrm{e}$ & $31.8 \mathrm{c}$ & $25.3 \mathrm{f}$ & $21.6 \mathrm{j}$ & $18,9 \mathrm{~L}$ & $20.5 \mathrm{i}$ & $23.1 \mathrm{j}$ & $19.6 n$ & $22.7 \mathrm{k}$ & $34.1 \mathrm{a}$ & $27.4 \mathrm{c}$ & 26.9cde \\
\hline 9-Rekafa & $32.8 \mathrm{~d}$ & $22.9 \mathrm{j}$ & $18.4 p$ & $20.2 \mathrm{k}$ & $18.8 \mathrm{~m}$ & $18.7 \mathrm{~m}$ & $19.6 \mathrm{j}$ & 19.30 & $20.1 \mathrm{~m}$ & $17.9 n$ & $22.5 \mathrm{~h}$ & $21.7 \mathrm{j}$ & $21.1 \mathrm{~h}$ \\
\hline 10-Jeous Q & $37.0 \mathrm{~b}$ & $31.0 \mathrm{~d}$ & $26.4 \mathrm{~g}$ & $28.1 \mathrm{~d}$ & $23.6 \mathrm{~g}$ & $20.8 \mathrm{j}$ & $27.9 \mathrm{e}$ & $23.7 \mathrm{i}$ & $24.4 \mathrm{i}$ & $26.0 \mathrm{f}$ & $27.1 \mathrm{~d}$ & $29.9 \mathrm{a}$ & $27.1 b c$ \\
\hline 11-Muhand & $31.6 \mathrm{e}$ & $27.6 \mathrm{f}$ & $18.5 \mathrm{o}$ & $20.7 \mathrm{j}$ & $19.3 \mathrm{~L}$ & $22.4 \mathrm{~g}$ & $31.3 \mathrm{~d}$ & $35.2 \mathrm{~b}$ & $28.0 \mathrm{f}$ & $32.5 \mathrm{a}$ & $18.0 \mathrm{~m}$ & $23.4 \mathrm{~g}$ & $25.7 \mathrm{~cd}$ \\
\hline 12-Farok Br. & $22.7 \mathrm{i}$ & $37.1 \mathrm{a}$ & $22.5 \mathrm{i}$ & $27.4 \mathrm{e}$ & $18.7 \mathrm{n}$ & $21.3 \mathrm{i}$ & $21.8 \mathrm{~g}$ & $26.2 \mathrm{~g}$ & $33.7 \mathrm{~b}$ & $19.3 \mathrm{~m}$ & 15.10 & $27.3 \mathrm{~d}$ & $24.4 \mathrm{~d}-\mathrm{g}$ \\
\hline 13-Khane Br. & $39.3 \mathrm{a}$ & $25.0 \mathrm{~h}$ & $19.4 \mathrm{~m}$ & $22.7 \mathrm{~h}$ & $29.7 \mathrm{~b}$ & $36.8 \mathrm{c}$ & $39.5 b$ & $36.2 \mathrm{a}$ & $28.5 \mathrm{e}$ & $13.7 \mathrm{o}$ & $17.3 n$ & $22.0 \mathrm{i}$ & $27.5 b c$ \\
\hline 14-Ashe jam & $21.9 \mathrm{j}$ & $32.6 \mathrm{~b}$ & $37.4 \mathrm{~b}$ & $28.6 \mathrm{~b}$ & $25.9 \mathrm{e}$ & $38.5 \mathrm{a}$ & $33.6 \mathrm{c}$ & $31.9 \mathrm{~d}$ & $32.6 \mathrm{c}$ & $21.6 \mathrm{~L}$ & $18.9 \mathrm{~L}$ & $20.7 \mathrm{~m}$ & $32.1 \mathrm{~b}$ \\
\hline 15-Bedara & $35.2 \mathrm{c}$ & $24.9 \mathrm{i}$ & $39.1 \mathrm{a}$ & $37.6 \mathrm{a}$ & $31.9 \mathrm{a}$ & $37.0 \mathrm{~b}$ & $39.8 \mathrm{a}$ & $34.3 \mathrm{c}$ & $38.4 \mathrm{a}$ & $28.4 \mathrm{~d}$ & $23.8 \mathrm{f}$ & $28.4 \mathrm{~b}$ & $33.2 \mathrm{a}$ \\
\hline 16-Ibrhem Br. & $17.3 p$ & $16.1 \mathrm{p}$ & $19.5 \mathrm{~L}$ & $16.8 \mathrm{p}$ & $13.0 \mathrm{o}$ & $15.3 n$ & $10.6 n$ & $11.4 p$ & 13.60 & $12.5 \mathrm{p}$ & $14.0 \mathrm{p}$ & $11.8 \mathrm{p}$ & $14.3 \mathrm{i}$ \\
\hline
\end{tabular}

Total alkalinity (TA) in $\mathrm{mg} / \mathrm{L}$ : In this work, (Table 15), the mean value of (TA) and standard error of mean values were ranged from $(122.50 \pm 19.309)$ to $(300.00 \pm 44.239) \mathrm{mg} / \mathrm{L}$. The total mean value of (TA) obtained in of Khabur River were within the safe level. According to (WHO ., 2012), the maximum permissible limit and desired limit for alkalinity in potable water is 200 and $600 \mathrm{mg} / \mathrm{L}$. From the results shown in (Table 11) .Alkalinity concentrations in the analyzed River water samples were ranged from (81 to 349$) \mathrm{mg} / \mathrm{l}$. The maximum concentration was observed at site Ashjame in August, The high concentration of (TA) due to the presence of bicarbonates, hydroxides and carbonates in the river water. While the minimum concentration was observed at site Jalke in February . All sites showed value within maximum safe levels by (WHO, 2012). (Table 15), observed a significant difference in the (TA) at the studied River water. They have a significant difference at $(\mathrm{p} \leq 0.05)$. This work are significantly minimum than those obtained by (Riedh ., 2020). 
Table 11 Monthly variation of Total alkalinity (TA) in $\mathrm{mg} / \mathbf{L}$ in sixteen locations

\begin{tabular}{|c|c|c|c|c|c|c|c|c|c|c|c|c|c|}
\hline $\begin{array}{l}\text { Months } \\
\text { Sites }\end{array}$ & Mar. & Apr & May. & Jun. & Jul. & Aug. & Sept. & Oct. & Nov. & Dec. & Jan. & Feb. & Average \\
\hline 1- Jalke & 1390 & 1410 & $116 p$ & $120 p$ & $127 \mathrm{o}$ & $135 n$ & $139 \mathrm{~m}$ & $141 p$ & $126 \mathrm{p}$ & $110 p$ & $95 p$ & $81 \mathrm{p}$ & $122 \mathrm{j}$ \\
\hline 2-Batefa & $146 n$ & $127 p$ & 1230 & $129 \mathrm{o}$ & $131 \mathrm{~m}$ & $125 \mathrm{o}$ & $131 p$ & $148 n$ & 1330 & 1180 & $105 n$ & $87 n$ & $148 \mathrm{ij}$ \\
\hline 3- Jamsmo & $163 \mathrm{j}$ & $173 \mathrm{i}$ & $158 \mathrm{n}$ & $135 n$ & $129 n$ & $136 \mathrm{~m}$ & 1360 & 1440 & $137 \mathrm{n}$ & $126 n$ & $99 \mathrm{o}$ & $84 \mathrm{o}$ & 135 hij \\
\hline 4-Aelol Q. & $147 \mathrm{~m}$ & $152 \mathrm{~m}$ & $173 \mathrm{k}$ & $142 \mathrm{~m}$ & $136 \mathrm{~L}$ & 139L & $138 n$ & $157 \mathrm{~L}$ & $140 \mathrm{~m}$ & $129 \mathrm{~m}$ & $116 \mathrm{~m}$ & $93 \mathrm{~m}$ & $138 \mathrm{~g}-\mathrm{j}$ \\
\hline 5-Dalal Br. & $158 \mathrm{k}$ & $148 n$ & $168 \mathrm{~m}$ & $150 \mathrm{k}$ & $141 \mathrm{k}$ & $147 \mathrm{k}$ & $140 \mathrm{~L}$ & $153 \mathrm{~m}$ & $142 \mathrm{~L}$ & $131 \mathrm{~L}$ & $121 \mathrm{~L}$ & $109 \mathrm{~L}$ & $142 \mathrm{f}-\mathrm{i}$ \\
\hline 6-Solaf & $163 \mathrm{j}$ & $17 \mathrm{j} 1$ & $182 \mathrm{j}$ & $164 j$ & $149 \mathrm{j}$ & $153 \mathrm{j}$ & $147 \mathrm{k}$ & $159 \mathrm{k}$ & $147 \mathrm{j}$ & $136 \mathrm{k}$ & $127 \mathrm{k}$ & $114 \mathrm{k}$ & $151 \mathrm{e}-\mathrm{h}$ \\
\hline 7- Jean & $171 \mathrm{~h}$ & $18 \mathrm{~h} 9$ & $169 \mathrm{~L}$ & $179 \mathrm{j}$ & $163 \mathrm{i}$ & $158 \mathrm{i}$ & $149 \mathrm{j}$ & $163 \mathrm{j}$ & $144 \mathrm{k}$ & $141 \mathrm{j}$ & $129 \mathrm{j}$ & $119 \mathrm{j}$ & $156 \mathrm{efg}$ \\
\hline 8-Hasnke & 149L & $162 \mathrm{k}$ & $184 \mathrm{i}$ & $188 \mathrm{i}$ & $167 \mathrm{~h}$ & $174 \mathrm{~h}$ & $163 i$ & $170 \mathrm{i}$ & $151 \mathrm{i}$ & $149 \mathrm{i}$ & $135 \mathrm{i}$ & $123 \mathrm{i}$ & $159 \mathrm{ef}$ \\
\hline 9-Rekafa & $164 \mathrm{i}$ & $154 \mathrm{~L}$ & $188 \mathrm{~h}$ & $194 \mathrm{~h}$ & $173 \mathrm{~g}$ & $182 \mathrm{~g}$ & $172 \mathrm{~h}$ & $175 \mathrm{~h}$ & $159 \mathrm{~h}$ & $177 \mathrm{~g}$ & $151 \mathrm{~h}$ & $127 \mathrm{~h}$ & $168 \mathrm{e}$ \\
\hline 10- Jeous Q. & $247 \mathrm{f}$ & $268 \mathrm{f}$ & $259 \mathrm{f}$ & $310 \mathrm{f}$ & $285 \mathrm{e}$ & $254 \mathrm{f}$ & $195 \mathrm{~g}$ & $211 \mathrm{~g}$ & $173 \mathrm{~g}$ & $169 \mathrm{~h}$ & $159 \mathrm{~g}$ & $131 \mathrm{~g}$ & $226 \mathrm{~d}$ \\
\hline $11-\mathrm{M}_{\mathrm{t}}$ & $283 b$ & $311 \mathrm{c}$ & $284 \mathrm{~d}$ & $326 \mathrm{c}$ & $295 \mathrm{~d}$ & $281 \mathrm{~d}$ & $217 \mathrm{f}$ & $231 \mathrm{f}$ & $199 \mathrm{f}$ & $221 \mathrm{e}$ & $174 \mathrm{f}$ & $139 \mathrm{f}$ & $246 b c$ \\
\hline 12-Farok B & $281 \mathrm{c}$ & $295 \mathrm{~d}$ & $311 \mathrm{c}$ & $315 \mathrm{e}$ & $319 \mathrm{c}$ & $275 \mathrm{e}$ & $242 \mathrm{e}$ & $253 \mathrm{e}$ & $206 \mathrm{e}$ & $218 \mathrm{f}$ & 191d & $152 \mathrm{e}$ & $254 b$ \\
\hline 13Khane $\mathrm{Br}$. & $261 \mathrm{e}$ & $293 e$ & $281 \mathrm{e}$ & $317 d$ & $285 \mathrm{e}$ & $311 \mathrm{c}$ & $285 b$ & $277 d$ & $223 c$ & $253 c$ & $186 \mathrm{e}$ & $163 \mathrm{c}$ & $261 \mathrm{~b}$ \\
\hline 14Ashe jam & $272 d$ & $316 b$ & $327 \mathrm{~b}$ & $336 b$ & $323 b$ & $349 a$ & $281 \mathrm{c}$ & $311 \mathrm{~b}$ & $263 b$ & $264 b$ & $205 b$ & $195 b$ & $286 a$ \\
\hline 15-Bedara & $296 a$ & $325 a$ & $331 \mathrm{a}$ & $342 a$ & $331 \mathrm{a}$ & $342 b$ & $315 a$ & $325 a$ & $277 \mathrm{a}$ & $281 \mathrm{a}$ & $224 a$ & $211 \mathrm{a}$ & $300 \mathrm{a}$ \\
\hline 16-Ibrahem Br. & $217 \mathrm{~g}$ & $222 \mathrm{~g}$ & $236 \mathrm{~g}$ & $261 \mathrm{~g}$ & $274 f$ & $281 d$ & $269 \mathrm{~d}$ & $284 \mathrm{c}$ & $216 \mathrm{~d}$ & $233 \mathrm{~d}$ & $196 \mathrm{c}$ & $158 \mathrm{~d}$ & $237 \mathrm{~cd}$ \\
\hline
\end{tabular}

Nitrates (NO3-): In this study the mean value of (NO3-) and standard error of mean values were ranged from ( 1.800 $\pm .7804)$ to $(6.633 \pm 1.4736) \mathrm{mg} / \mathrm{L}$, (Table 15),. The mean value of (NO3-), were found within the safe level value of (WHO., 2011) which is $45 \mathrm{mg} / \mathrm{L}$. (Table 15) shows a significant differences of the nitrates at the studied river samples (Table 12) shows the value of nitrate was observed in the range of (o.8 $\mathrm{mg} / \mathrm{l}$ to 8.7$) \mathrm{mg} / \mathrm{L}$, the maximum

Table 12 Monthly Variation of Nitrates (NO3-) in mg/L in sixteen locations

\begin{tabular}{|c|c|c|c|c|c|c|c|c|c|c|c|c|c|}
\hline $\begin{array}{ll} & \text { Months } \\
\text { Sites } & \end{array}$ & Mar. & Apr & May. & Jun & Jul. & Aug. & Sept. & Oct. & Nov. & Dec. & Jan. & Feb. & Average \\
\hline 1-Jalke & $1.8 \mathrm{o}$ & $1.6 \mathrm{o}$ & $2.0 \mathrm{n}$ & $1.9 \mathrm{k}$ & $1.4 \mathrm{~L}$ & $1.1 \mathrm{~m}$ & $0.8 \mathrm{p}$ & $1.0 \mathrm{p}$ & $1.6 \mathrm{p}$ & $2.8 \mathrm{n}$ & $3.6 n$ & $2.0 \mathrm{p}$ & $1.8 \mathrm{i}$ \\
\hline 2-Batefa & $2.2 n$ & $1.8 \mathrm{n}$ & $2.6 \mathrm{~m}$ & $1.4 \mathrm{~m}$ & $1.7 \mathrm{j}$ & $1.0 \mathrm{n}$ & $1.1 \mathrm{o}$ & $1.6 n$ & $1.9 \mathrm{o}$ & $2.5 \mathrm{o}$ & $3.5 \mathrm{o}$ & $2.5 \mathrm{o}$ & 1.9 ih \\
\hline 3-Jamsemo & $2.9 \mathrm{~m}$ & $1.6 \mathrm{o}$ & $3.2 \mathrm{~L}$ & $1.7 \mathrm{~L}$ & $1.2 \mathrm{~m}$ & $1.5 \mathrm{~L}$ & $1.7 \mathrm{~m}$ & $1.3 \mathrm{o}$ & $2.3 n$ & $3.7 \mathrm{~L}$ & $3.9 \mathrm{~m}$ & $2.7 \mathrm{n}$ & 2.3 ghi \\
\hline 4-Aelol Q & $3.3 \mathrm{~L}$ & $1.9 \mathrm{~m}$ & $3.7 \mathrm{k}$ & $1.9 \mathrm{k}$ & $1.6 \mathrm{k}$ & $1.7 \mathrm{k}$ & $1.6 \mathrm{n}$ & $1.9 \mathrm{~m}$ & $2.6 \mathrm{~m}$ & $3.3 \mathrm{~m}$ & $2.7 \mathrm{p}$ & $3.3 \mathrm{~m}$ & 2.4gh \\
\hline 5-Dalal 1 & $4.0 \mathrm{j}$ & $2.6 \mathrm{~L}$ & $3.9 \mathrm{j}$ & $2.0 \mathrm{j}$ & $1.9 \mathrm{i}$ & $2.0 \mathrm{j}$ & $1.9 \mathrm{~L}$ & $2.1 \mathrm{~L}$ & $2.9 \mathrm{~L}$ & $2.8 \mathrm{n}$ & $4.2 \mathrm{~L}$ & $3.6 \mathrm{~L}$ & $2.8 \mathrm{~g}$ \\
\hline 6-Dalal 2 & $4,4 \mathrm{i}$ & $3.7 \mathrm{j}$ & $4.2 \mathrm{i}$ & $2.5 \mathrm{i}$ & $2.2 \mathrm{~h}$ & $2.7 \mathrm{i}$ & $2.0 \mathrm{k}$ & $2.6 \mathrm{k}$ & $3.5 \mathrm{k}$ & $4.3 \mathrm{k}$ & $5.1 \mathrm{k}$ & $4.0 \mathrm{k}$ & $3.4 \mathrm{f}$ \\
\hline 7-Jean & $4.9 \mathrm{~h}$ & $2.9 \mathrm{k}$ & $4.9 \mathrm{~g}$ & $2.9 \mathrm{~g}$ & $3.9 \mathrm{e}$ & $4.9 \mathrm{~g}$ & $3.6 \mathrm{j}$ & $2.9 \mathrm{j}$ & $3.9 \mathrm{j}$ & $4.8 \mathrm{j}$ & $6.3 \mathrm{j}$ & $5.8 \mathrm{~h}$ & $4.3 \mathrm{e}$ \\
\hline 8-Hasanke & $5.1 \mathrm{~g}$ & $3.8 \mathrm{i}$ & $5.0 \mathrm{f}$ & $2.5 \mathrm{i}$ & $4.6 \mathrm{c}$ & $5.2 \mathrm{f}$ & $4.3 \mathrm{i}$ & $3.7 \mathrm{~h}$ & $4.3 \mathrm{i}$ & $5.3 \mathrm{i}$ & $6.8 \mathrm{i}$ & $4.9 \mathrm{j}$ & 4.6de \\
\hline 9-Rekafa & $5.6 \mathrm{f}$ & $5.1 \mathrm{~g}$ & $4.2 \mathrm{i}$ & $3.1 \mathrm{e}$ & $3.6 \mathrm{f}$ & $6.0 \mathrm{~d}$ & $4.8 \mathrm{~g}$ & $3.3 \mathrm{i}$ & $5.2 \mathrm{~h}$ & $6.1 \mathrm{~h}$ & $7.3 \mathrm{~g}$ & $5.3 \mathrm{i}$ & $4.9 \mathrm{~d}$ \\
\hline 10-Jeous Q & $6.0 \mathrm{~d}$ & $6.4 \mathrm{e}$ & $4.8 \mathrm{~h}$ & $3.5 \mathrm{~d}$ & $4.2 \mathrm{f}$ & $6.8 \mathrm{~b}$ & $4.6 \mathrm{~h}$ & $4.4 \mathrm{~g}$ & $5.8 \mathrm{f}$ & $6.7 \mathrm{f}$ & $8.0 \mathrm{e}$ & $6.2 \mathrm{~g}$ & $5.6 \mathrm{bc}$ \\
\hline 11-Muhand & $6.5 \mathrm{~b}$ & $5.9 \mathrm{f}$ & $5.3 \mathrm{~d}$ & $2.8 \mathrm{~h}$ & $3.9 \mathrm{e}$ & $5.3 \mathrm{e}$ & $5.7 \mathrm{e}$ & $6.1 \mathrm{f}$ & $5.3 \mathrm{~g}$ & $6.9 \mathrm{e}$ & $8.2 \mathrm{c}$ & $7.1 \mathrm{e}$ & $5.7 \mathrm{~b}$ \\
\hline 12-Farok Br. & $5.8 \mathrm{e}$ & $6.6 \mathrm{~d}$ & $5.9 \mathrm{c}$ & $3.9 \mathrm{~b}$ & $5.1 \mathrm{a}$ & $6.6 \mathrm{c}$ & $5.9 \mathrm{~d}$ & $6.8 \mathrm{~d}$ & $6.7 \mathrm{c}$ & $7.3 \mathrm{c}$ & $8.1 \mathrm{~d}$ & $7.7 \mathrm{c}$ & $6.3 \mathrm{a}$ \\
\hline 13-Khane $\mathrm{Br}$. & $4.9 \mathrm{~h}$ & $6.9 \mathrm{~b}$ & $6.1 \mathrm{a}$ & $4.0 \mathrm{a}$ & $4.8 \mathrm{~b}$ & $7.2 \mathrm{a}$ & $6.7 \mathrm{~b}$ & $7.3 \mathrm{c}$ & $6.4 \mathrm{~d}$ & $7.1 \mathrm{~d}$ & $7.9 \mathrm{f}$ & $8.3 a$ & $6.4 \mathrm{a}$ \\
\hline 14-Ashe jam & $6.3 \mathrm{c}$ & $7.3 \mathrm{a}$ & $5.9 \mathrm{c}$ & $3.5 \mathrm{~d}$ & $3.9 \mathrm{e}$ & $5.3 \mathrm{e}$ & $6.4 \mathrm{c}$ & $7.7 \mathrm{~b}$ & $7.0 \mathrm{~b}$ & $8.0 \mathrm{a}$ & $8.7 \mathrm{a}$ & $8.0 \mathrm{~b}$ & $6.5 \mathrm{a}$ \\
\hline 15-Bedara & $6.9 \mathrm{a}$ & $6.7 \mathrm{c}$ & $6.0 \mathrm{~b}$ & $3.0 \mathrm{f}$ & $5.1 \mathrm{a}$ & $6.0 \mathrm{~d}$ & $7.1 \mathrm{a}$ & $8.1 \mathrm{a}$ & $7.6 \mathrm{a}$ & $7.4 \mathrm{~b}$ & $8.4 \mathrm{~b}$ & $7.3 \mathrm{~d}$ & $6.6 \mathrm{a}$ \\
\hline 16- Ibrahem Br. & $6.7 \mathrm{c}$ & $6.6 \mathrm{c}$ & $6.5 \mathrm{~b}$ & $2.7 \mathrm{~h}$ & $5.2 \mathrm{a}$ & $6.6 \mathrm{c}$ & $6.7 \mathrm{~b}$ & $7.8 \mathrm{~b}$ & $6.5 \mathrm{~d}$ & $7.2 \mathrm{~d}$ & $8.2 \mathrm{c}$ & $8.2 a$ & $6.5 \mathrm{a}$ \\
\hline
\end{tabular}

Sulfate $\left(\mathrm{SO}_{4}{ }^{2-}\right)$ : In this work the mean value of $\left(\mathrm{SO}_{4}{ }^{2-}\right)$ and standard error of mean values was ranged from ( $16.00 \pm 4.918)$ to $(70.50 \pm 12.881) \mathrm{mg} / \mathrm{L}$ (Table 15$)$. The mean value of $\left(\mathrm{SO}_{4}{ }^{2-}\right)$ were found within the permissible limit. The higher safe level of sulfate value in water is $400 \mathrm{mg} / \mathrm{l}$ according to (WHO ., 2011). (Table 13), the river water samples is varying from ( 9 to 95) $\mathrm{mg} / \mathrm{L}$. A higher concentration of sulfate was observed at site Bedara in January, the maximum value due to discharge concentration was observed at site Ashejame in January, The major sources of (NO3-), value in water might be anthropogenic or from the utilize of fertilizer on agricultural land. While the lower concentration was observed at site Jalke in September. They have a significant differences at $(\mathrm{p} \leq 0.05)$ (Table 15) . Same results obtained by (Yumin et al .2019 from domestic waste and untreated sewage, while the minimum concentration of sulfate was recorded at site Jalke in September. The sulfate concentration of all locations lies within the safe levels according to (WHO ., 2012) $(250 \mathrm{mg} / \mathrm{L})$. (Table15), shows a significant variation in the $\left(\mathrm{SO}_{4}{ }^{2-}\right)$ at the studied River water. They have a significant difference at $(\mathrm{p} \leq$ $0.05)$. Table 13 Monthly variation of Sulfate $\left(\mathrm{SO}_{4}{ }^{2-}\right)$ in $\mathrm{mg} / \mathrm{L}$ in sixteen location

Table 13 Monthly variation of Sulfate $\left(\mathbf{S O}_{4}{ }^{2-}\right)$ in $\mathrm{mg} / \mathrm{L}$ in sixteen locations

\begin{tabular}{|l|l|l|l|l|l|l|l|l|l|l|l|l|l|}
\hline $\begin{array}{l}\text { Months } \\
\text { Sites }\end{array}$ & Mar. & Apr & May. & Jun. & Jul. & Aug. & Sept. & Oct. & Nov. & Dec. & Jan. & Feb. & Average \\
\hline 1-Jalke & $19 \mathrm{~m}$ & $17 \mathrm{n}$ & $21 \mathrm{o}$ & $15 \mathrm{n}$ & $13 \mathrm{o}$ & $11 \mathrm{p}$ & $9 \mathrm{p}$ & $11 \mathrm{n}$ & $13 \mathrm{n}$ & $18 \mathrm{p}$ & $26 \mathrm{o}$ & $19 \mathrm{n}$ & $16 \mathrm{i}$ \\
\hline 2-Batefa & $13 \mathrm{o}$ & $14 \mathrm{p}$ & $26 \mathrm{n}$ & $17 \mathrm{~m}$ & $11 \mathrm{p}$ & $15 \mathrm{n}$ & $13 \mathrm{o}$ & $13 \mathrm{~m}$ & $10 \mathrm{o}$ & $22 \mathrm{o}$ & $31 \mathrm{~m}$ & $26 \mathrm{~L}$ & $17 \mathrm{i}$ \\
\hline 3-Jamsemo & $19 \mathrm{~m}$ & $16 \mathrm{o}$ & $33 \mathrm{k}$ & $13 \mathrm{o}$ & $15 \mathrm{n}$ & $13 \mathrm{o}$ & $18 \mathrm{c}$ & $10 \mathrm{o}$ & $16 \mathrm{~L}$ & $28 \mathrm{~m}$ & $28 \mathrm{n}$ & $21 \mathrm{~m}$ & $19 \mathrm{ih}$ \\
\hline 4-Aelol Q & $16 \mathrm{n}$ & $18 \mathrm{~m}$ & $28 \mathrm{~m}$ & $18 \mathrm{~L}$ & $19 \mathrm{~L}$ & $17 \mathrm{~m}$ & $14 \mathrm{n}$ & $16 \mathrm{~L}$ & $14 \mathrm{~m}$ & $25 \mathrm{n}$ & $24 \mathrm{p}$ & $37 \mathrm{j}$ & $20 \mathrm{ih}$ \\
\hline
\end{tabular}




\begin{tabular}{|l|l|l|l|l|l|l|l|l|l|l|l|l|l|}
\hline 5-Dalal 1 & $21 \mathrm{~L}$ & $19 \mathrm{~L}$ & $38 \mathrm{j}$ & $21 \mathrm{j}$ & $17 \mathrm{~m}$ & $19 \mathrm{~L}$ & $16 \mathrm{~m}$ & $13 \mathrm{~m}$ & $17 \mathrm{k}$ & $37 \mathrm{k}$ & $42 \mathrm{~L}$ & $31 \mathrm{k}$ & $22 \mathrm{gh}$ \\
\hline 6-Dalal 2 & $22 \mathrm{k}$ & $20 \mathrm{k}$ & $31 \mathrm{~L}$ & $19 \mathrm{k}$ & $21 \mathrm{k}$ & $26 \mathrm{k}$ & $31 \mathrm{k}$ & $25 \mathrm{k}$ & $20 \mathrm{j}$ & $31 \mathrm{~L}$ & $55 \mathrm{j}$ & $47 \mathrm{i}$ & $29 \mathrm{~g}$ \\
\hline 7-Jean & $32 \mathrm{~g}$ & $25 \mathrm{j}$ & $46 \mathrm{i}$ & $27 \mathrm{~h}$ & $25 \mathrm{j}$ & $37 \mathrm{j}$ & $39 \mathrm{j}$ & $29 \mathrm{j}$ & $27 \mathrm{i}$ & $39 \mathrm{j}$ & $48 \mathrm{k}$ & $51 \mathrm{~h}$ & $35 \mathrm{f}$ \\
\hline 8-Hasanke & $39 \mathrm{e}$ & $28 \mathrm{i}$ & $38 \mathrm{j}$ & $24 \mathrm{i}$ & $31 \mathrm{i}$ & $41 \mathrm{i}$ & $44 \mathrm{i}$ & $34 \mathrm{i}$ & $41 \mathrm{~h}$ & $52 \mathrm{i}$ & $61 \mathrm{i}$ & $56 \mathrm{~g}$ & $40 \mathrm{e}$ \\
\hline 9-Rekafa & $41 \mathrm{~d}$ & $33 \mathrm{~h}$ & $52 \mathrm{~h}$ & $29 \mathrm{~g}$ & $39 \mathrm{~h}$ & $45 \mathrm{~h}$ & $53 \mathrm{~h}$ & $38 \mathrm{~h}$ & $49 \mathrm{~g}$ & $58 \mathrm{~h}$ & $74 \mathrm{f}$ & $62 \mathrm{f}$ & $47 \mathrm{~d}$ \\
\hline 10-Jeous Q & $36 \mathrm{f}$ & $39 \mathrm{~g}$ & $61 \mathrm{c}$ & $54 \mathrm{~d}$ & $52 \mathrm{~g}$ & $61 \mathrm{~g}$ & $59 \mathrm{~g}$ & $41 \mathrm{~g}$ & $55 \mathrm{e}$ & $62 \mathrm{f}$ & $68 \mathrm{~h}$ & $68 \mathrm{e}$ & $54 \mathrm{c}$ \\
\hline 11-Muhand & $47 \mathrm{c}$ & $42 \mathrm{e}$ & $58 \mathrm{~d}$ & $69 \mathrm{a}$ & $59 \mathrm{e}$ & $68 \mathrm{e}$ & $73 \mathrm{~d}$ & $47 \mathrm{f}$ & $52 \mathrm{f}$ & $69 \mathrm{~d}$ & $84 \mathrm{~d}$ & $77 \mathrm{c}$ & $62 \mathrm{~b}$ \\
\hline 12-Farok Br & $31 \mathrm{~h}$ & $48 \mathrm{~d}$ & $53 \mathrm{~g}$ & $51 \mathrm{e}$ & $68 \mathrm{~d}$ & $71 \mathrm{~d}$ & $69 \mathrm{e}$ & $52 \mathrm{e}$ & $59 \mathrm{~d}$ & $63 \mathrm{e}$ & $71 \mathrm{~g}$ & $81 \mathrm{a}$ & $59 \mathrm{bc}$ \\
\hline 13-Khane Br & $48 \mathrm{~b}$ & $51 \mathrm{c}$ & $62 \mathrm{~b}$ & $64 \mathrm{~b}$ & $73 \mathrm{c}$ & $83 \mathrm{~b}$ & $78 \mathrm{~b}$ & $59 \mathrm{c}$ & $63 \mathrm{c}$ & $74 \mathrm{c}$ & $88 \mathrm{~b}$ & $79 \mathrm{~b}$ & $68 \mathrm{a}$ \\
\hline 14-Ashe jam & $51 \mathrm{a}$ & $61 \mathrm{a}$ & $56 \mathrm{e}$ & $57 \mathrm{c}$ & $79 \mathrm{~b}$ & $94 \mathrm{a}$ & $75 \mathrm{c}$ & $66 \mathrm{~b}$ & $69 \mathrm{~b}$ & $79 \mathrm{~b}$ & $85 \mathrm{c}$ & $74 \mathrm{~d}$ & $70 \mathrm{a}$ \\
\hline 15- Bedara & $47 \mathrm{c}$ & $32 \mathrm{~h}$ & $38 \mathrm{j}$ & $29 \mathrm{~g}$ & $31 \mathrm{j}$ & $71 \mathrm{~d}$ & $78 \mathrm{~b}$ & $47 \mathrm{f}$ & $56 \mathrm{e}$ & $62 \mathrm{f}$ & $74 \mathrm{f}$ & $68 \mathrm{e}$ & $71 \mathrm{a}$ \\
\hline 16- Ibrahem Br. & $41 \mathrm{~d}$ & $39 \mathrm{~g}$ & $58 \mathrm{~d}$ & $65 \mathrm{~b}$ & $52 \mathrm{~g}$ & $41 \mathrm{j}$ & $59 \mathrm{j}$ & $41 \mathrm{~g}$ & $59 \mathrm{~d}$ & $62 \mathrm{f}$ & $71 \mathrm{~g}$ & $62 \mathrm{f}$ & $40 \mathrm{e}$ \\
\hline
\end{tabular}

Table 14 WQI values for Khabur River during studied period

\begin{tabular}{|c|c|c|c|c|c|c|c|c|c|c|c|c|c|c|c|c|}
\hline $\begin{array}{l}\text { Cana } \\
\text { dian } \\
\text { WQI }\end{array}$ & Jalke & $\begin{array}{l}\text { Batef } \\
\text { a }\end{array}$ & $\begin{array}{l}\text { Jams } \\
\text { emo }\end{array}$ & $\begin{array}{l}\text { Aelol } \\
\text { Q. }\end{array}$ & $\begin{array}{l}\text { Dalal } \\
\mathrm{Br}\end{array}$ & Solaf & $\begin{array}{l}\text { Jea } \\
n\end{array}$ & $\begin{array}{l}\text { Hasa } \\
\text { nake }\end{array}$ & $\begin{array}{l}\text { Rek } \\
\text { afa. }\end{array}$ & $\begin{array}{l}\text { Jeou } \\
\text { sq. }\end{array}$ & $\begin{array}{l}\text { Muh } \\
\text { and. }\end{array}$ & $\begin{array}{l}\text { Far } \\
\text { ok } \\
\text { Q. }\end{array}$ & $\begin{array}{l}\mathrm{Ka} \\
\text { ne } \\
\mathrm{B}\end{array}$ & $\begin{array}{l}\text { As } \\
\text { he } \\
\text { Ja } \\
\text { m }\end{array}$ & $\begin{array}{l}\text { Beda } \\
\text { ra }\end{array}$ & $\begin{array}{l}\text { Brahe } \\
\text { mbr }\end{array}$ \\
\hline $\begin{array}{l}\text { Drink } \\
\text { ing }\end{array}$ & $\begin{array}{l}\text { Excel } \\
\text { lent }\end{array}$ & $\begin{array}{l}\text { Excel } \\
\text { lent }\end{array}$ & $\begin{array}{l}\text { Excel } \\
\text { lent }\end{array}$ & Good & Good & Poor & $\begin{array}{l}\text { Po } \\
\text { or }\end{array}$ & Poor & Poor & $\begin{array}{l}\text { Poo } \\
\text { r } \\
\end{array}$ & Poor & $\begin{array}{l}\text { Po } \\
\text { or }\end{array}$ & $\begin{array}{l}\text { Po } \\
\text { or }\end{array}$ & $\begin{array}{l}\text { Po } \\
\text { or }\end{array}$ & Poor & Poor \\
\hline $\begin{array}{l}\text { Irriga } \\
\text { tion }\end{array}$ & $\begin{array}{l}\text { Excel } \\
\text { lent }\end{array}$ & $\begin{array}{l}\text { Excel } \\
\text { lent }\end{array}$ & $\begin{array}{l}\text { Excel } \\
\text { lent }\end{array}$ & $\begin{array}{l}\text { Excel } \\
\text { lent }\end{array}$ & $\begin{array}{l}\text { Excel } \\
\text { lent }\end{array}$ & $\begin{array}{l}\text { Excel } \\
\text { lent }\end{array}$ & $\begin{array}{l}\text { Go } \\
\text { od }\end{array}$ & Good & $\begin{array}{l}\text { Goo } \\
\text { d }\end{array}$ & $\begin{array}{l}\text { Goo } \\
\mathrm{d}\end{array}$ & Good & $\begin{array}{l}\text { Go } \\
\text { od }\end{array}$ & $\begin{array}{l}\text { Go } \\
\text { od }\end{array}$ & $\begin{array}{l}\text { Go } \\
\text { od }\end{array}$ & $\begin{array}{l}\text { Excel } \\
\text { lent }\end{array}$ & Good \\
\hline
\end{tabular}

Table 15: Physical-chemical properties of Khabur River, data represented as mean \pm S.D, during studied period.

\begin{tabular}{|c|c|c|c|c|c|c|c|c|c|c|c|c|c|}
\hline Sites & $\mathrm{T}^{\circ} \mathrm{C}$ & EC & TDS & $\mathrm{pH}$ & DO & BOD & TH & Ca2+ & Mg2+ & $\mathrm{Cl}$ & TA & NO3- & $\left(\mathrm{SO}_{4}{ }^{2-}\right)$ \\
\hline Jalke & $\begin{array}{l}12.07 \pm 3.4 \\
\mathrm{a}\end{array}$ & $\begin{array}{l}446.7 \pm 3 \\
6.5^{\mathrm{e}}\end{array}$ & $\begin{array}{l}285.4 \pm 2 \\
3.5^{\mathrm{e}}\end{array}$ & $\begin{array}{l}8.06 \pm \\
0.1^{\text {ab }}\end{array}$ & $\begin{array}{l}8.9 \pm 0.67 \\
\mathrm{ab}\end{array}$ & $2.55 \pm 0.9 \mathrm{i}$ & $\begin{array}{l}434.5 \pm 1 \\
1.8 \mathrm{k}\end{array}$ & $\begin{array}{l}350.5 \pm 3 \\
31.8 \mathrm{~g}\end{array}$ & $\begin{array}{l}84.0 \pm 225.9 \\
h\end{array}$ & $\begin{array}{l}21.8 \pm 2.2 \\
9 \mathrm{~h}\end{array}$ & $122.8 \pm 19.3 \mathrm{j}$ & $\begin{array}{l}1.8 \pm 0.78 \\
\mathrm{i}\end{array}$ & $\begin{array}{l}2.72 \pm 0.9 \\
7 \mathrm{i}\end{array}$ \\
\hline Batefa & $\begin{array}{l}12.12 \pm 3.3 \\
1 \mathrm{a}\end{array}$ & $\begin{array}{l}452.4 \pm 3 \\
7.1 \mathrm{e}\end{array}$ & $\begin{array}{l}289.1 \pm 0 . \\
23 \mathrm{e}\end{array}$ & $\begin{array}{l}8.1 \pm 0.20 \\
5 \mathrm{a}\end{array}$ & $\begin{array}{c}8.94 \\
\pm 0.95 \mathrm{a}\end{array}$ & $\begin{array}{l}3.00 \\
\pm 0.79 \mathrm{hi}\end{array}$ & $\begin{array}{l}343.7 \pm 1 \\
3.3 \mathrm{k}\end{array}$ & $\begin{array}{l}321.0 \\
\pm 36.6 \mathrm{~h}\end{array}$ & $\begin{array}{l}118.0 \pm 41.2 \\
\text { def }\end{array}$ & $\begin{array}{l}34.9 \pm 11 . \\
3 \mathrm{~h}\end{array}$ & $\begin{array}{l}125.2 \pm 16.5 \\
\text { ih }\end{array}$ & $\begin{array}{l}1.98 \pm 0.7 \\
\text { lih }\end{array}$ & $\begin{array}{l}17.5 \pm 6.9 \\
0 \mathrm{i}\end{array}$ \\
\hline Jamsem & $12.1 \pm 3.3$ & $\begin{array}{l}453.8 \pm 3 \\
1.9 \mathrm{e}\end{array}$ & $\begin{array}{l}302.9 \pm 4 \\
1.51 \mathrm{e}\end{array}$ & $\begin{array}{l}8.01 \pm 0.1 \\
3 \mathrm{bc}\end{array}$ & $\begin{array}{l}8.8 \pm 0.75 \\
a b\end{array}$ & $\begin{array}{l}3.44 \pm 0.91 \mathrm{~g} \\
\mathrm{hi}\end{array}$ & $\begin{array}{l}442.7 \pm 1 \\
1.7 \mathrm{k}\end{array}$ & $\begin{array}{l}361.6 \pm 1 \\
5.3 \mathrm{f}\end{array}$ & $80.2 \pm 7.4 \mathrm{~h}$ & $\begin{array}{l}22.6 \pm 3.2 \\
\text { fgh }\end{array}$ & $\begin{array}{l}135.0 \pm 24.7 \\
\text { hij }\end{array}$ & $\begin{array}{l}2.30 \pm 0.9 \\
3 \mathrm{ghi}\end{array}$ & $\begin{array}{l}19.1 \pm 7.0 \\
2 \mathrm{ih}\end{array}$ \\
\hline AlelolQ & $\begin{array}{l}17.9 \pm 4.31 \\
\mathrm{a}\end{array}$ & $\begin{array}{l}463.2 \pm 2 \\
8.9 \mathrm{e}\end{array}$ & $\begin{array}{l}296.0 \pm 2 \\
5.0 \mathrm{e}\end{array}$ & $\begin{array}{l}8.0 \pm 0.22 \\
\text { b }\end{array}$ & $\begin{array}{l}8.7 \pm 0.58 \\
\mathrm{ab}\end{array}$ & $3.65 \pm 1.0 \mathrm{gh}$ & $\begin{array}{l}474.0 \pm 3 \\
7.3 \mathrm{j}\end{array}$ & $\begin{array}{l}368.5 \pm 2 \\
2.1 \mathrm{f}\end{array}$ & $\begin{array}{l}114.4 \pm 55.2 \\
\text { fgh }\end{array}$ & $\begin{array}{l}36.4 \pm 21 \\
5 \mathrm{gh}\end{array}$ & $\begin{array}{l}138.5 \pm 20.1 \\
\mathrm{gj}\end{array}$ & $\begin{array}{l}2.45 \pm 0.7 \\
8 \mathrm{gh}\end{array}$ & $\begin{array}{l}20.5 \pm 6.8 \\
2 \mathrm{ih}\end{array}$ \\
\hline Dala B & $\begin{array}{l}20.8 \pm 1.43 \\
\mathrm{a}\end{array}$ & $\begin{array}{l}480.1 \pm 4 \\
6.5 \mathrm{e}\end{array}$ & $\begin{array}{l}305.2 \pm 3 \\
2.0 \mathrm{~d}\end{array}$ & $\begin{array}{l}7.9 \pm 0.19 \\
\text { bc }\end{array}$ & $\begin{array}{l}8.7 \pm 0.62 \\
\mathrm{ab}\end{array}$ & $3.7 \pm 0.67 \mathrm{gh}$ & $\begin{array}{l}484.0 \pm 3 \\
0.6 \mathrm{ij}\end{array}$ & $\begin{array}{l}372.0 \pm 2 \\
7.4 \mathrm{e}\end{array}$ & $\begin{array}{l}111.0 \pm 22.5 \\
\text { fgh }\end{array}$ & $\begin{array}{l}24.5 \pm 4.0 \\
\text { def }\end{array}$ & $\begin{array}{l}142.3 \pm 16.0 \\
\text { fi }\end{array}$ & $\begin{array}{l}2.82 \pm 0.8 \\
8 \mathrm{~g}\end{array}$ & $\begin{array}{l}24.2 \pm 3.9 \\
\text { gh }\end{array}$ \\
\hline Solaf & $24.8 \pm 2.1 \mathrm{a}$ & $\begin{array}{l}519.9 \pm 5 \\
6.6 \mathrm{~d}\end{array}$ & $\begin{array}{l}332.3 \pm 3 \\
6.4 \mathrm{~d}\end{array}$ & $\begin{array}{l}7.92 \pm 0.6 \\
5 \text { bcd }\end{array}$ & $\begin{array}{l}8.6 \pm 0.64 \\
\text { b }\end{array}$ & $4.39 \pm 0.42 \mathrm{~g}$ & $\begin{array}{l}501.6 \pm 4 \\
4.7 \mathrm{hi}\end{array}$ & $\begin{array}{l}410.5 \pm 1 \\
4.5 \mathrm{~d}\end{array}$ & $\begin{array}{l}87.6 \pm 14.5 \mathrm{~g} \\
\mathrm{~h}\end{array}$ & $\begin{array}{l}26.4 \pm 4.8 \\
\text { cd }\end{array}$ & $\begin{array}{l}151.0 \pm 18.9 \\
\text { eh }\end{array}$ & $\begin{array}{l}3.43 \pm 1.0 \\
\mathrm{f}\end{array}$ & $\begin{array}{l}29.0 \pm 11 . \\
3 \mathrm{~g}\end{array}$ \\
\hline Jean & $12.6 \pm 3.3 \mathrm{a}$ & $\begin{array}{l}523.2 \pm 6 \\
3.3 \mathrm{~d}\end{array}$ & $\begin{array}{l}334.4 \pm 4 \\
0.6 \mathrm{~d}\end{array}$ & $\begin{array}{l}7.8 \pm 0.25 \\
\text { cde }\end{array}$ & $\begin{array}{l}8.3 \pm 0.53 \\
\mathrm{c}\end{array}$ & $5.64 \pm 1.4 \mathrm{f}$ & $\begin{array}{l}517.1 \pm 5 \\
1.3 \mathrm{gh}\end{array}$ & $\begin{array}{l}424.5 \pm 1 \\
1.1 \mathrm{c}\end{array}$ & $\begin{array}{l}97.3 \pm 10.4 \mathrm{f} \\
\text { gh }\end{array}$ & $\begin{array}{l}22.9 \pm 4.4 \\
\mathrm{~g}\end{array}$ & $\begin{array}{l}156.1 \pm 20.5 \\
\text { efg }\end{array}$ & $\begin{array}{l}4.30 \pm 1.1 \\
\mathrm{e}\end{array}$ & $\begin{array}{l}35.4 \pm 9.3 \\
\text { f }\end{array}$ \\
\hline Hasanke & $12.8 \pm 3.3 \mathrm{a}$ & $\begin{array}{l}542 \pm 76 . \\
8\end{array}$ & $\begin{array}{l}346.6 \pm 4 \\
9.1 \mathrm{c}\end{array}$ & $\begin{array}{l}7.8 \pm 0.22 \\
\text { efg }\end{array}$ & $\begin{array}{l}8.2 \pm 0.50 \\
\mathrm{~cd}\end{array}$ & $9.1 \pm 2.6 \mathrm{e}$ & $\begin{array}{l}534.3 \pm 5 \\
3.9 \mathrm{fg}\end{array}$ & $\begin{array}{l}432.1 \pm 1 \\
1.8 \mathrm{c}\end{array}$ & $\begin{array}{l}112.2 \pm 22.6 \\
\mathrm{fg}\end{array}$ & $\begin{array}{l}39.3 \pm 11 \\
4 \text { cde }\end{array}$ & $\begin{array}{l}159.5 \pm 19.1 \\
\text { ef }\end{array}$ & $\begin{array}{l}4.62 \pm 1.0 \\
\text { de }\end{array}$ & $\begin{array}{l}40.7 \pm 11 . \\
1 \mathrm{e}\end{array}$ \\
\hline Rekafa & $13.1 \pm 3.4 \mathrm{a}$ & $\begin{array}{l}586.0 \pm 1 \\
11.6 \mathrm{~d}\end{array}$ & $\begin{array}{l}376.6 \pm 6 \\
8.5 \mathrm{bc}\end{array}$ & $\begin{array}{l}7.72 \pm 0.2 \\
6 \text { efg }\end{array}$ & $\begin{array}{l}7.9 \pm 0.54 \\
\text { de }\end{array}$ & $12.3 \pm 3.6 \mathrm{~d}$ & $\begin{array}{l}567.7 \pm 5 \\
3.4 \mathrm{e}\end{array}$ & $\begin{array}{l}426.0 \pm 1 \\
5.5 \mathrm{c}\end{array}$ & $\begin{array}{l}137.3 \pm 54.8 \\
\text { cde }\end{array}$ & $\begin{array}{l}21.0 \pm 4.0 \\
\mathrm{~h}\end{array}$ & $\begin{array}{l}168.0 \pm 18.3 \\
\text { e }\end{array}$ & $\begin{array}{l}4.96 \pm 1.2 \\
\mathrm{~d}\end{array}$ & $\begin{array}{l}47.7 \pm 12 . \\
9 \mathrm{~d}\end{array}$ \\
\hline JeousQ & $11.9 \pm 4.4 \mathrm{a}$ & $\begin{array}{l}586.1 \pm 8 \\
9.4 \mathrm{c}\end{array}$ & $\begin{array}{l}387.7 \pm 5 \\
6.8 \mathrm{bc}\end{array}$ & $\begin{array}{l}7.8 \pm 0.25 \\
\text { ghi }\end{array}$ & $\begin{array}{l}6.9 \pm 0.70 \\
\text { de }\end{array}$ & $15.1 \pm 3.17 \mathrm{~b}$ & $\begin{array}{l}573.5 \pm 3 \\
9.9 \mathrm{de}\end{array}$ & $\begin{array}{l}425.1 \pm 1 \\
1.5 \mathrm{c}\end{array}$ & $\begin{array}{l}148.4 \pm 46.7 \\
\text { bc }\end{array}$ & $\begin{array}{l}27.1 \pm 4.2 \\
\text { bc }\end{array}$ & $\begin{array}{l}221.7 \pm 56.5 \\
\mathrm{~d}\end{array}$ & $\begin{array}{l}5.6 \pm 1.3 \mathrm{~b} \\
\mathrm{c}\end{array}$ & $\begin{array}{l}54.6 \pm 10 . \\
8 \mathrm{c}\end{array}$ \\
\hline Muhand & $13.5 \pm 3.4 \mathrm{a}$ & $\begin{array}{l}629.3 \pm 1 \\
05.0 \mathrm{c}\end{array}$ & $\begin{array}{l}387.2 \pm 8 \\
4.2 \mathrm{bc}\end{array}$ & $\begin{array}{l}7.6 \pm 0.26 \\
\text { fg }\end{array}$ & $\begin{array}{l}7.7 \pm 0.62 \\
\text { ef }\end{array}$ & $\begin{array}{l}15.6 \pm 1.98 \\
\mathrm{ab}\end{array}$ & $\begin{array}{l}593.0 \pm 5 \\
2.5 \mathrm{~cd}\end{array}$ & $\begin{array}{l}431.6 \pm 1 \\
0.5 \mathrm{c}\end{array}$ & $\begin{array}{l}161.4 \pm 57.3 \\
\text { bc }\end{array}$ & $\begin{array}{l}25.7 \pm 6.0 \\
\text { cd }\end{array}$ & $\begin{array}{l}246.7 \pm 58.4 \\
\text { bc }\end{array}$ & $5.7 \pm 1.4 \mathrm{~b}$ & $\begin{array}{l}62.0 \pm 13 . \\
2 \mathrm{~b}\end{array}$ \\
\hline Farok B & $\begin{array}{l}13.59 \pm 3.4 \\
6 \mathrm{a}\end{array}$ & $\begin{array}{l}639.5 \pm 1 \\
05.7 \mathrm{~b}\end{array}$ & $\begin{array}{l}401.4 \pm 7 \\
1.5 \mathrm{abc}\end{array}$ & $\begin{array}{l}7.7 \pm 0.2 \\
\mathrm{hi}\end{array}$ & $\begin{array}{l}7.77 \pm 0.5 \\
\text { ef }\end{array}$ & $16.6 \pm 2.9 \mathrm{a}$ & $\begin{array}{l}594.4 \pm 4 \\
1.5 \mathrm{~cd}\end{array}$ & $\begin{array}{l}451.2 \pm 1 \\
1.5 \mathrm{~b}\end{array}$ & $\begin{array}{l}143.1 \pm 42.9 \\
\text { dcd }\end{array}$ & $\begin{array}{l}24.4 \pm 6.2 \\
\mathrm{dg}\end{array}$ & $\begin{array}{l}254.8 \pm 54.0 \\
\text { b }\end{array}$ & $\begin{array}{l}6.3 \pm 61.1 \\
\mathrm{a}\end{array}$ & $\begin{array}{l}59.7 \pm 13 . \\
5 \mathrm{bc}\end{array}$ \\
\hline Khane B & $13.5 \pm 3.4 \mathrm{a}$ & $\begin{array}{l}658.0 \pm 9 \\
6.5 \mathrm{~b}\end{array}$ & $\begin{array}{l}413.5 \pm 6 \\
2.1 \mathrm{ab}\end{array}$ & $\begin{array}{l}7.6 \pm 0.2 \\
\mathrm{hi}\end{array}$ & $\begin{array}{l}7.5 \pm 0.77 \\
\text { fg }\end{array}$ & $16.3 \pm 2.6 \mathrm{a}$ & $\begin{array}{l}608.5 \pm 4 \\
8.3 \mathrm{bc}\end{array}$ & $\begin{array}{l}472.0 \pm 1 \\
2.3 \mathrm{a}\end{array}$ & $\begin{array}{l}136.5 \pm 55.4 \\
\text { cde }\end{array}$ & $\begin{array}{l}27.5 \pm 8.8 \\
\text { bc }\end{array}$ & $\begin{array}{l}26182 \pm 47.8 \\
\text { b }\end{array}$ & $\begin{array}{l}66.3 \pm 1.3 \\
\text { a }\end{array}$ & $\begin{array}{l}68.5 \pm 12 . \\
6 \mathrm{a}\end{array}$ \\
\hline Ashejam & $13.8 \pm 3.6 \mathrm{a}$ & $\begin{array}{l}675.0 \pm 9 \\
1.2 \mathrm{a}\end{array}$ & $\begin{array}{l}424.0 \pm 6 \\
6.4 \mathrm{a}\end{array}$ & $\begin{array}{l}7.5 \pm 0.2 \mathrm{~g} \\
\mathrm{~h}\end{array}$ & $7.5 \pm 0.6 \mathrm{~g}$ & $16.3 \pm 1.9 \mathrm{a}$ & $\begin{array}{l}617.7 \pm 4 \\
4.4 \mathrm{ab}\end{array}$ & $\begin{array}{l}429.0 \pm 2 \\
1.4 \mathrm{c}\end{array}$ & $\begin{array}{l}188.7 \pm 55.4 \\
\mathrm{ab}\end{array}$ & $\begin{array}{l}28.6 \pm 6.7 \\
\mathrm{~b}\end{array}$ & $\begin{array}{l}286.8 \pm 49.6 \\
\mathrm{a}\end{array}$ & $6.5 \pm 1.6 \mathrm{a}$ & $\begin{array}{l}70.5 \pm 12 . \\
9 \mathrm{a}\end{array}$ \\
\hline Bedara & $13.6 \pm 3.3 \mathrm{a}$ & $\begin{array}{l}659.7 \pm 8 \\
8.1 \mathrm{~b}\end{array}$ & $\begin{array}{l}22.0 \pm 6 \\
5.6 \mathrm{a}\end{array}$ & $7.5 \pm 0.2 \mathrm{ij}$ & $\begin{array}{l}7.3 \pm 0.6 \\
\mathrm{~cd}\end{array}$ & $15.7 \pm 2.3 \mathrm{ab}$ & $\begin{array}{l}636.0 \pm 5 \\
4.1 \mathrm{a}\end{array}$ & $\begin{array}{l}469.7 \pm 1 \\
1.4 \mathrm{a}\end{array}$ & $\begin{array}{l}166.3 \pm 59.7 \\
\text { bc }\end{array}$ & $\begin{array}{l}33.2 \pm 5.6 \\
\mathrm{a}\end{array}$ & $300.0 \pm 442 \mathrm{a}$ & $6.6 \pm 1.4 \mathrm{a}$ & $\begin{array}{l}69.1 \pm 17 . \\
4 \mathrm{a}\end{array}$ \\
\hline $\begin{array}{l}\text { Ibrahem } \\
\text { B }\end{array}$ & $12.8 \pm 3.1 \mathrm{a}$ & $\begin{array}{l}649.0 \pm 8 \\
3.0 \mathrm{~b}\end{array}$ & $\begin{array}{l}391.0 \pm 5 \\
1.6 \mathrm{~b}\end{array}$ & $\begin{array}{l}7.8 \pm 0.1 \\
\mathrm{jk}\end{array}$ & $\begin{array}{l}8.0 \pm 0.5 \\
\mathrm{~cd}\end{array}$ & $13.8 \pm 3.4 \mathrm{c}$ & $\begin{array}{l}546.9 \pm 5 \\
8.7 \mathrm{f}\end{array}$ & $\begin{array}{l}401.0 \pm 1 \\
7.3 \mathrm{~d}\end{array}$ & $\begin{array}{l}154.2 \pm 66.0 \\
b c\end{array}$ & $\begin{array}{l}14.3 \pm 2.7 \\
\mathrm{i}\end{array}$ & $\begin{array}{l}237.2 \pm 38.2 \\
\text { cd }\end{array}$ & $\begin{array}{l}5.1 \pm 1.2 \mathrm{c} \\
\mathrm{d}\end{array}$ & $\begin{array}{l}56.8 \pm 13 . \\
7 \mathrm{bc}\end{array}$ \\
\hline Total & $14.4 \pm 1.3 \mathrm{a}$ & $\begin{array}{l}560.2 \pm 1 \\
10.8 \mathrm{~b}\end{array}$ & $\begin{array}{l}355.9 \pm 7 \\
1.5 \mathrm{ab}\end{array}$ & $\begin{array}{l}7.8 \pm 0.2 \\
\text { def }\end{array}$ & $\begin{array}{l}8.2 \pm 0.7 \mathrm{c} \\
\mathrm{d}\end{array}$ & $9.8 \pm 6.0 \mathrm{c}$ & $\begin{array}{l}536.0 \pm 7 \\
80 \mathrm{f}\end{array}$ & $\begin{array}{l}409.1 \pm 4 \\
6.8 \mathrm{~d}\end{array}$ & $\begin{array}{l}127.5 \pm 62.8 \\
b c\end{array}$ & $\begin{array}{l}26.9 \pm 11 . \\
2 \mathrm{i}\end{array}$ & $\begin{array}{l}194.1 \pm 69.5 \\
\text { cd }\end{array}$ & $\begin{array}{l}4.4 \pm 2.0 \mathrm{c} \\
\mathrm{d}\end{array}$ & $\begin{array}{l}43.2 \pm 22 . \\
4 \mathrm{bc}\end{array}$ \\
\hline
\end{tabular}

Note: Values in each rows with different letters are significantly different at $\mathbf{P}<0.05$. Values in rows with same letters are

not significantly different

\section{CONCLUSIONS}

The present study has an attempt to evaluate the contamination impact on the river water related with the discharge of untreated wastewater from the "Zakho city" in Khabur River. This experiment confirmed that some chemical and physical parameters like $\mathrm{pH}$, temperature and dissolved oxygen, chloride , and total alkalinity met the standard acceptable limit according to (WHO., 2012).While Total hardness varied from $(535.02 \pm 78.014) \mathrm{mg} / \mathrm{l}$. Calcium (Ca 2+) varied from (409.19 \pm 46.875) $\mathrm{mg} / \mathrm{l}$. Magnesium $(\mathrm{Mg} 2+)$,were exceed the permissible limit compared to ( WHO.,2014). The results suggested that the water quality of Khabur River is degraded to downstream and might be impacted by agriculture and domestic sewage water from Zakho city. There is need to ensure that wastewater is properly treated before discharge into the Khabur River. 


\section{REFERENCES}

APHA (American Public Health Association) . (2017). Standard methods for the examination of water and wastewater.23rd ed., Washington, DC, USA. pp 22831

Ayoub, M. B., Yousif, Kamil M. 2015. Water Quality Index of the little Khabur River in Zakho city Kurdistan Region. The 7th Intern . Conf. on Sustainable Development in Building and Environ.-SuDBE2015, Reading, UK, Ref. No. 3005

Ackson Adiyiah Nyantakyi, Bernard Fei- Baffoe, Osei Akoto, 2020. Seasonal Variations in Physicochemical and Nutrient Water Quality of River Tano in Ghana. International Journal of Environmental Chemistry.Volume 4, Issue 1, June 2020, Pages: $1-1$

Bishnu Kant Shukla1 , Pushpendra Kumar Sharma2* and Mohd Asif Khan3 2021. Physico-Chemical Study of Some Surface Water Bodies of Punjab Journal of Physics: Conference $\begin{array}{lllll}\text { Series } & 1531 & \text { (2020) } & 012121 & \text { IOP Publishing }\end{array}$ doi:10.1088/1742-6596/1531/1/012121

Bilyaminu Garba Jega, O. O. Adebisi S. S. Manga A. Muhamma 2020. Vol 1 No 3 (2020) Physicochemical Properties of Tagangu Seasonal River Receiving Abattoir Wastewater Discharge, Aliero, Kebbi State, NigeriaVol 1 No 3 (2020)

MohdYawar Ali Khan 2021, Evaluation of physicochemical and heavy metals characteristics in surface water under anthropogenic activities using multivariate statistical methods, Garra River, Ganges Basin, India .Envion Eng Res > Volume 26(6); 2021

Musher Rashed Ahmed 2015. Influence of sewage water on the water quality of Khabor river in Zakho city at the second international conference on ecology, environment and energy ICEEE2015

Kamil . 2016 effectiveness of chlorine treatment on physicochemical charaterisics of water supplies for Zakho city Kurdistan of
Iraq,International Journal of Current Research Vol. 8, Issue, 10, pp.40319-40324, October, 2016

Lilia Zaou .2020 . Assessment of water quality bounamoussa river in el tarf region using water quality index (Algeria) International Physical Medicine \& Rehabilitation Journal .Volume 4 Issue $6-2020$.

NidhiGupta ${ }^{a}$ PankajPandey ${ }^{\mathrm{a}}$ JakirHussain 2017. Effect of physicochemical and biological parameters on the quality of river water of Narmada, Madhya Pradesh, Indi . Volume 31, Issue 1, April 2017, Pages 11-23

O. I. Solana1*, F. A. Omotola1 ,G. B. Ogungbayi1 , O. T. Opafola 2020 Quantification of metals, physicochemical and microbiological properties of consumed sachet/surface waters in Ayetoro Community, Ogun State, Nigeria . J. Mater. Environ. Sci., 2020, Volume 11, Issue 6, Page 856867

Riedh Abass Abdul- jabbar 2020 . Pollution treatments of Tigris River through the chemical and physical characterization in Waset Governorate .Eurasian Journal of Biosciences, 2020 Volume 14 Issue 2, pp. 4409-4414

Saurabh Mishra ${ }^{1,3}$, Amit Kumar 2021 .Estimation of physicochemical characteristics and associated metal contaminationrisk in river Narmada, India . Environ Eng Res > Volume 26(1); 2021

WHO (2012)., Guidelines for Drinking-water Quality., Second edition, Addendum to 2, Health Criteria and Other Supporting Information

WHO (2011)., Guidelines for Drinking-water Quality., Second edition, Addendum to 2, Health Criteria and Other Supporting Information

Yumin Wang, Ran Yu, and Guangcan Zhu 2019.Evaluation of Physicochemical Characteristics in Drinking Water Sources Emphasized on Fluoride: A Case Study of Yancheng, ChinaInt J Environ Res Public Health v.16(6); 2019 Mar. 